Supporting Information for

\title{
Mechanism of Catalytic Aziridination with Manganese Corrole: The Often Postulated High- Valent Mn(V) Imido is not the Group Transfer Reagent
}

Michael J. Zdilla and Mahdi M. Abu-Omar

Brown Laboratory, Department of Chemistry, Purdue University, 560 Oval Drive, West

Lafayette, IN 47907 


\section{Table of Contents}

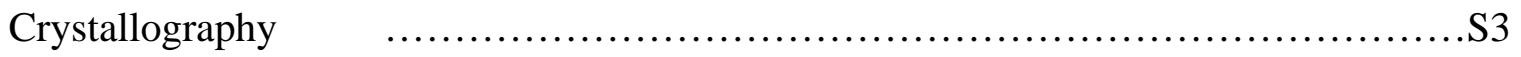

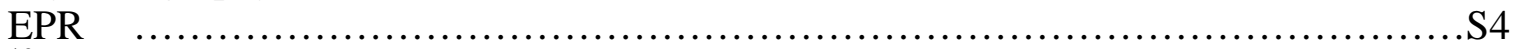

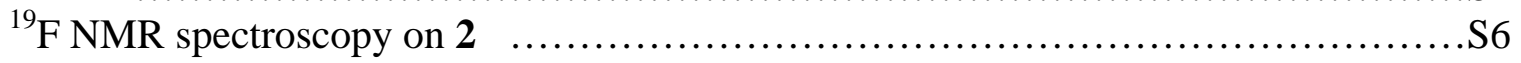

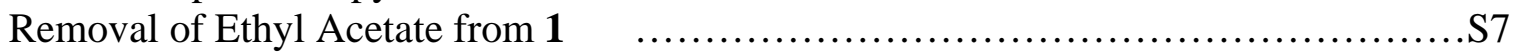

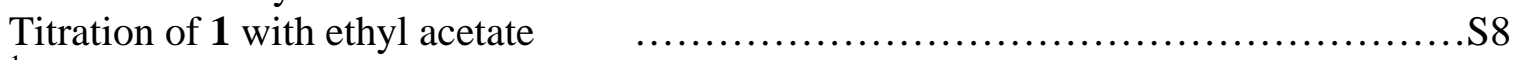

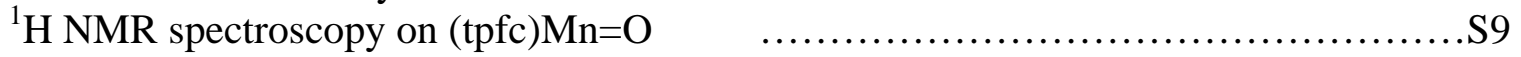

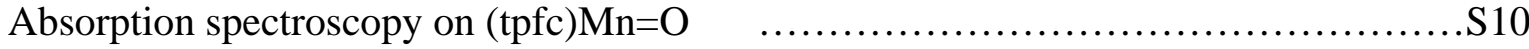

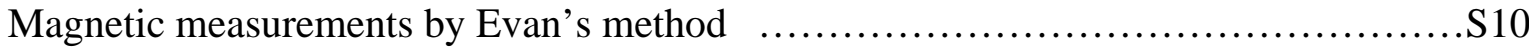

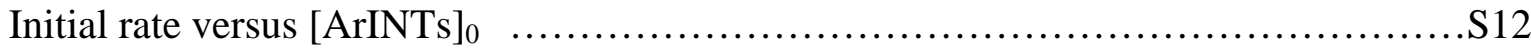

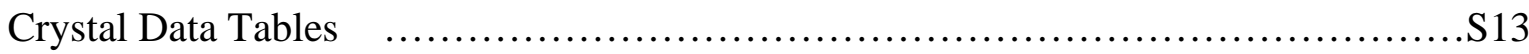

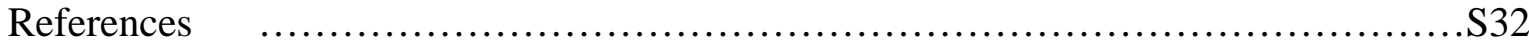

Appendix: Derivation........................................................... 


\section{Crystallography}

Crystal structures were obtained on a Nonius Kappa CCD single crystal X-ray diffractometer.

Samples were mounted on a glass fiber with mineral oil, and data were collected at $150 \mathrm{~K}$.

Data were integrated using Denzo scalepack, and solution/refinement was performed using ShelX 97.

Crystal Structure of $\mathbf{M n}(\mathbf{t p f c})($ EtOAc):EtOAc (1). Crystals of 1 were grown by vapor diffusion at $-20^{\circ} \mathrm{C}$ in a double vial apparatus. $\mathbf{1}$ in EtOAc in an inner vial was stored in an outer vial containing $n$-heptane. After several weeks, large green rods were observed.
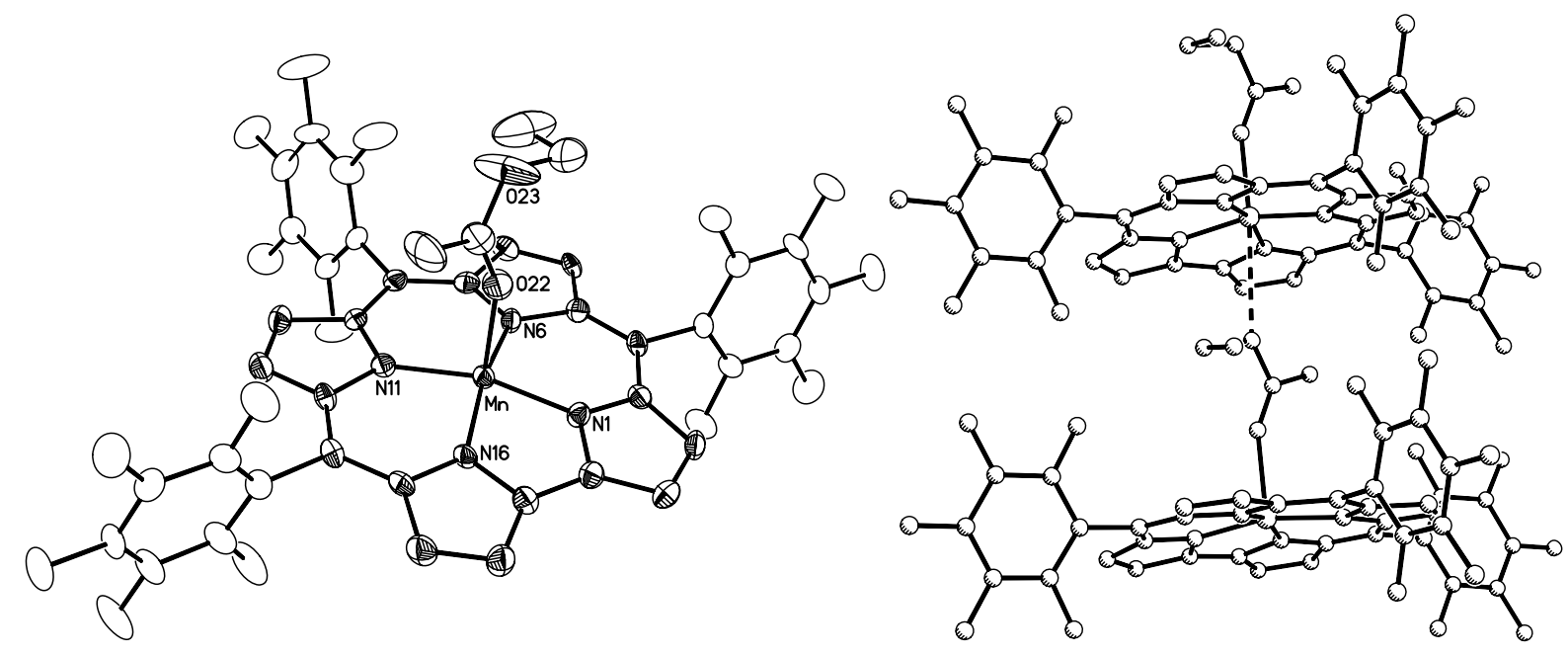

Figure S1. Left: Structure of Mn(tpfc)(EtOAc)·EtOAc (1) with thermal ellipsoids (50\%).

Pentafluorophenyl atoms are designated by boundary ellipses, and axial-ligand carbon atoms are designated by boundary and principle ellipses. Hydrogen atoms are not shown. Right: Structure of Mn(tpfc)(EtOAc)·EtOAc (1) showing interaction of axial ethyl acetate ligand with neighboring manganese atom $3.15 \AA$ away.

Crystal Structure of (tpfc)CrNTs. $80 \mathrm{mg}(80 \mu \mathrm{mol})$ of $\mathrm{Cr}(\mathrm{tpfc})(\mathrm{py}){ }_{2}{ }^{1}$ was dissolved in 1.5 $\mathrm{mL} \mathrm{C}_{6} \mathrm{H}_{6}$, and $89 \mathrm{mg}(240 \mu \mathrm{mol})$ of PhINTs was added. The mixture was stirred to afford a 
red solution. The solution was decanted from the undissolved PhINTs, and dried in vacuo.

The mixture was dissolved in $1 \mathrm{~mL} \mathrm{Et}_{2} \mathrm{O}$, and chilled at $-30{ }^{\circ} \mathrm{C}$ to afford dark red crystals (50\%). These were filtered and dried. Large single crystals were grown by vapor diffusion at $20{ }^{\circ} \mathrm{C}$ in a double vial apparatus. A solution of (tpfc)CrNTs in $\mathrm{Et}_{2} \mathrm{O}$ in an inner vial was stored in an outer vial containing $n$-pentane at $-30^{\circ} \mathrm{C}$. After a week, single large red needles were obtained.
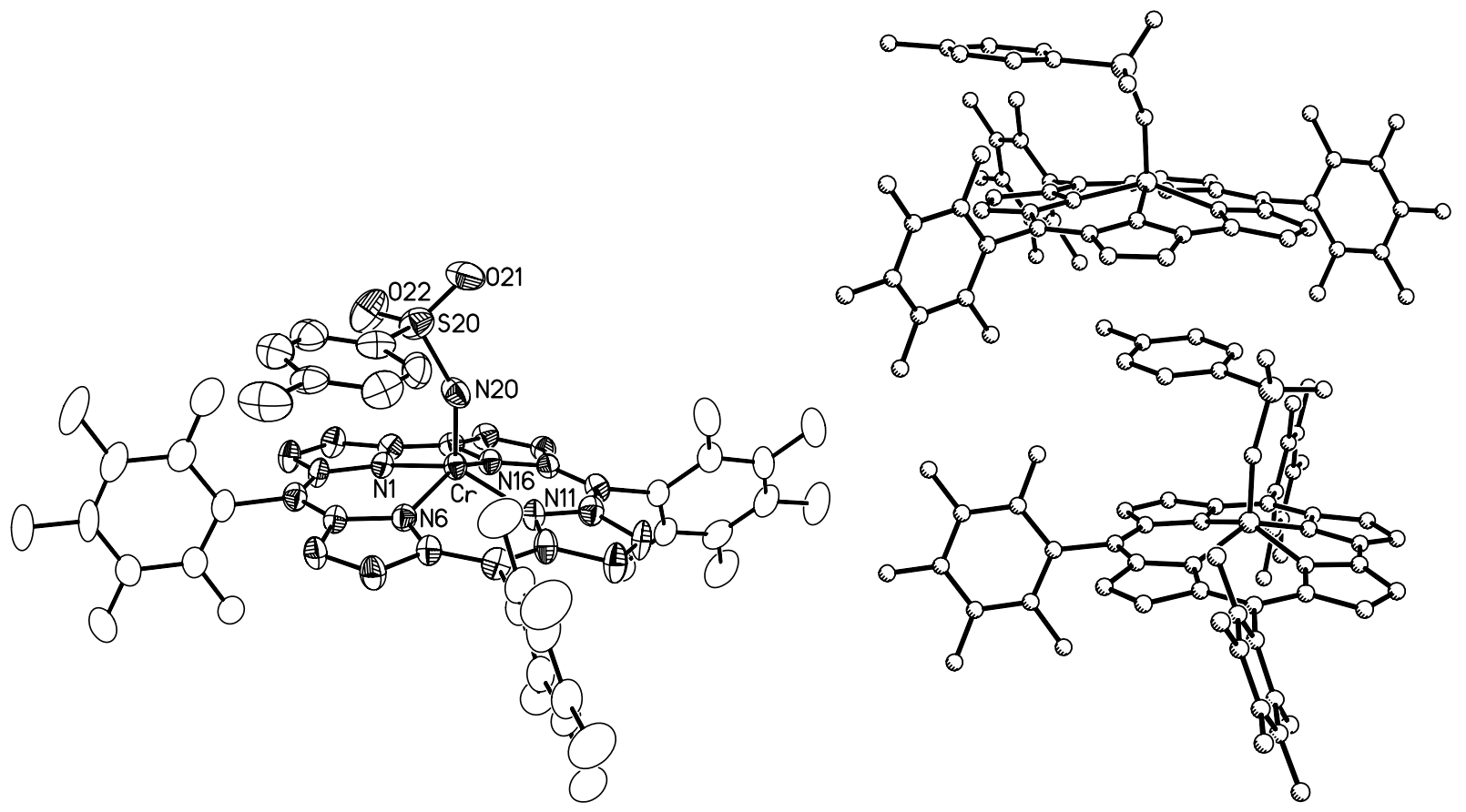

Figure S2. Left: Structure of (tpfc)CrNTs with thermal ellipsoids (50\%). Pentafluorophenyl atoms are designated by boundary ellipses, and axial-ligand carbon atoms are designated by boundary and principle ellipses. Hydrogen atoms are not shown. Right: Structure of (tpfc)CrNTs showing $\pi$-stacking interaction of axial tosylimido ligand with neighboring metal corrole.

EPR of 2. X-band EPR spectroscopy was carried out on 2 at $5 \mathrm{~K}$. The spectrum exhibits 4 distinct signals. One at $g=8$, assigned to residual $\mathrm{Mn}(\mathrm{III})$, one at $g=4.3$, assigned to $\mathrm{Mn}$ (II) 
under high zero-field-splitting, one at $g=4$, assigned to 2 , and one at $g=2$, assigned to a spin $1 / 2$ manganese dimer, a minor product from hydrolysis (Figure S3, top). After addition of $\mathrm{H}_{2} \mathrm{O}$, the hydrolysis signal at $g=2$ intensifies, the signal for 2 at $g=4$, and the signals for residual Mn(II) and Mn(III) do not change (Fig. S3, bottom).
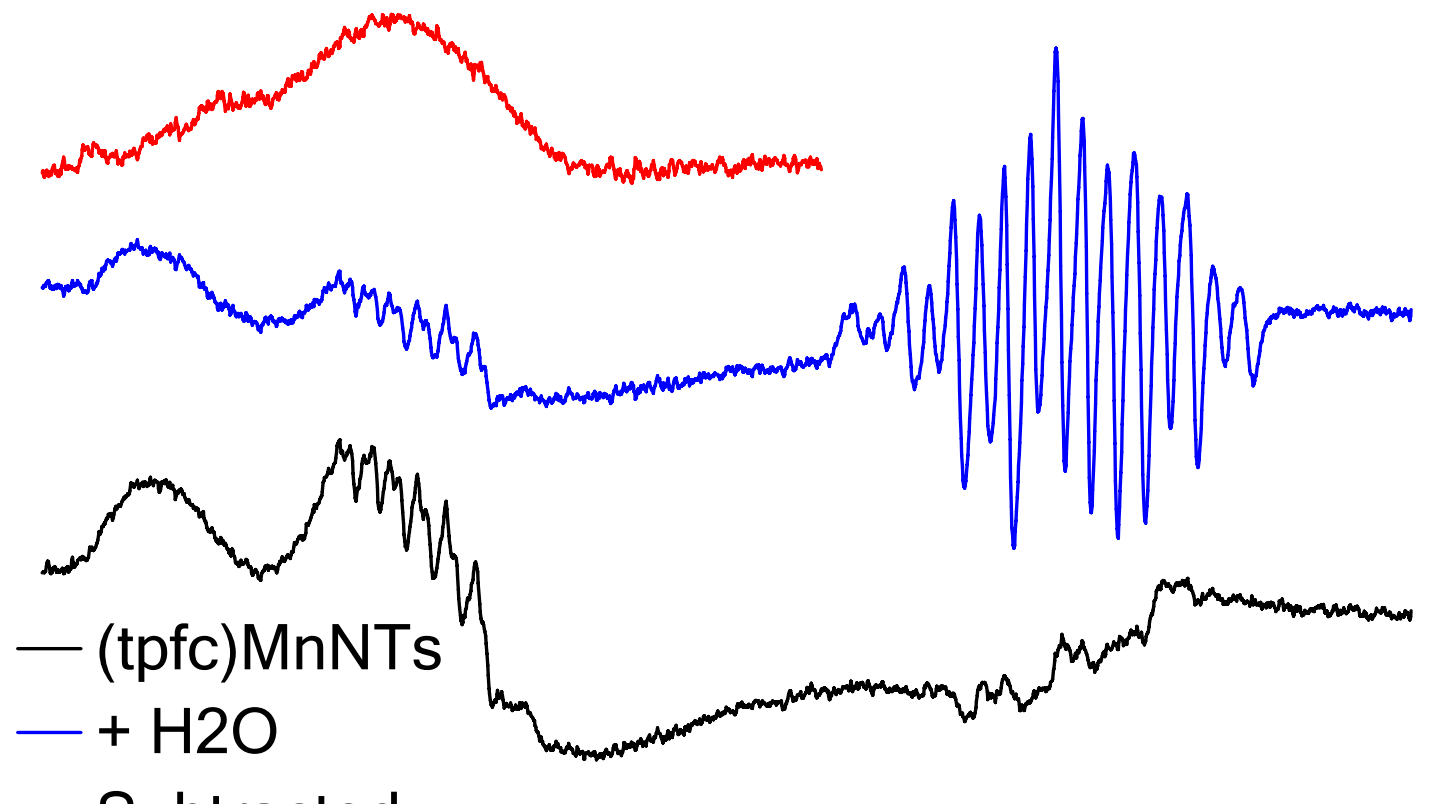

- Subtracted

\section{0 \\ 1500 \\ 2500 \\ 3500 \\ 4500 \\ B (G)}

Figure S3. X-Band EPR spectrum of in situ generated 2 at $5 \mathrm{~K}$ in dry toluene (bottom), after addition of $\mathrm{H}_{2} \mathrm{O}$ (middle), and the subtraction of the two spectra to remove the hyperfine feature at $g=4.3$, displaying the $g=4$ signal assigned to 2 (top). MW freq. $9.44 \mathrm{GHz}$, MW Power 1.0 mW, Mod Freq. 100 kHz, Mod Amp. 14.33 G. 
EPR of (tpfc)CrNTs. X-band EPR spectroscopy was carried out on (tpfc)CrNTs at room temperature and 90K. The spectrum shows an isotropic $\mathrm{S}=1 / 2$ signal at $g=1.984$ with hyperfine splitting visible at room temperature.

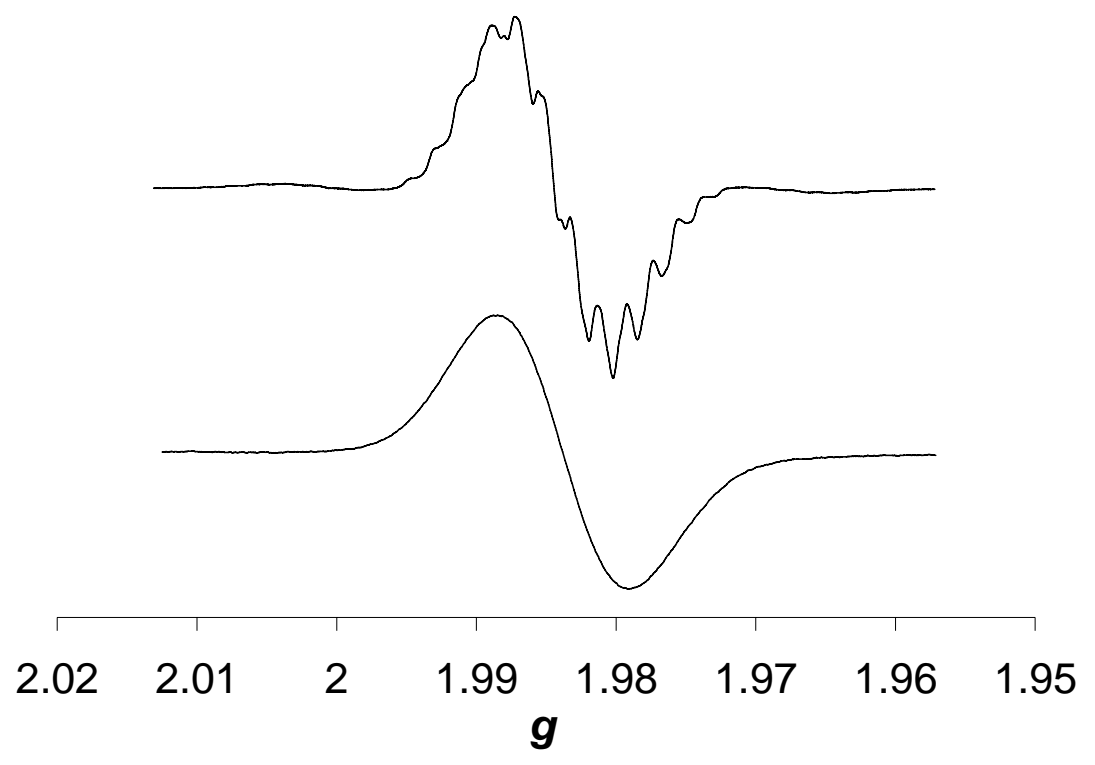

Figure S4. X-Band EPR spectrum at room temperature (top) and $90 \mathrm{~K}$ (bottom). MW freq. 9.78 GHz, MW Power 10.0 mW, Mod Freq. 100 kHz, Mod Amp. 7.083 G.

${ }^{19}$ F NMR spectroscopy was carried out on 2 by stirring 1 (6.9 mg, $\left.6.7 \mu \mathrm{mol}\right)$ with ArINTs (4.0 mg, 8.1 $\mu \mathrm{mol})$ in $\mathrm{CDCl}_{3}(5 \mathrm{~mL})$. The resulting red-brown solution was analyzed by NMR. ${ }^{19} \mathrm{~F}$ NMR (CD $\left.{ }_{3} \mathrm{CN}\right): \delta$-196.6 (br), -171.3 (v br), -174.5 (br), -175.6 (br), -178 (v br). 


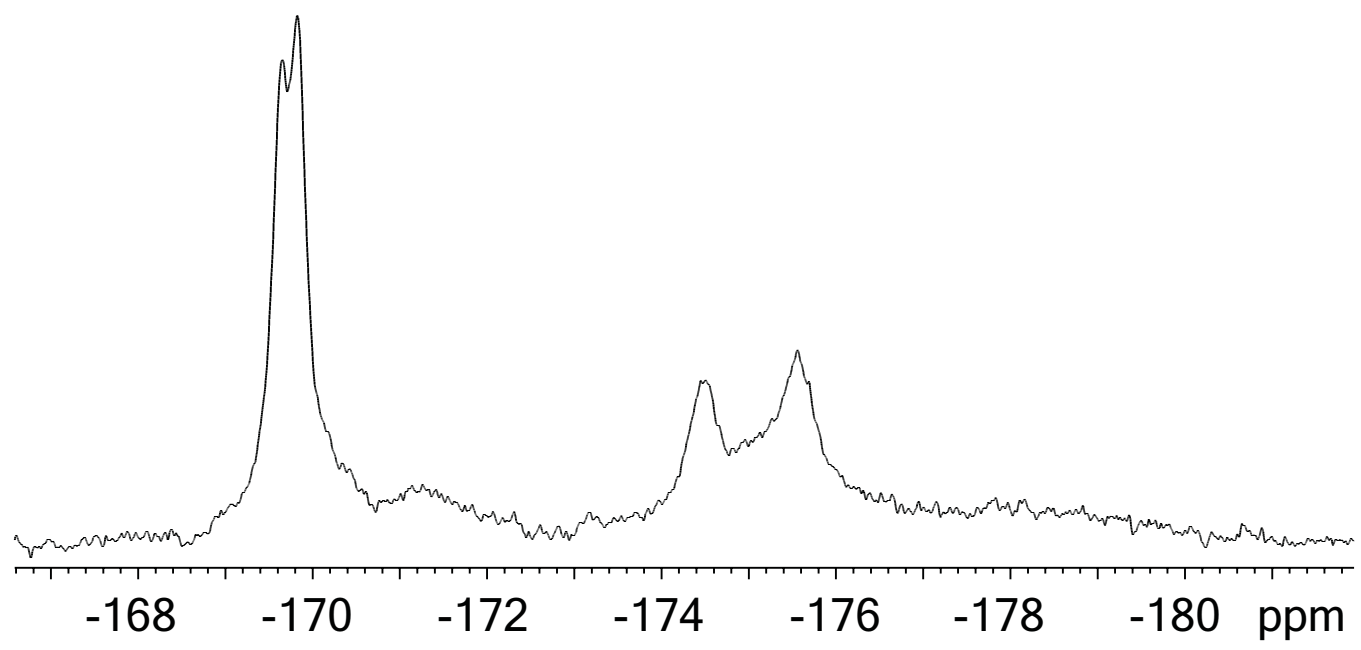

Figure S5. ${ }^{19} \mathrm{~F}$ NMR of 2 generated in situ in $\mathrm{CD}_{3} \mathrm{CN}$.

\section{Removal of Ethyl Acetate from 1}

Ethyl acetate was removed from $\mathbf{1}$ by sublimation. $\mathbf{1}$ was heated under vacuum in a schlenk tube. Some material sublimed and deposited on the sides of the tube, while some remained on the heated bottom of the tube. The absorption spectra of both solids were identical to that of crystalline 1(EtOAc)·EtOAc. The removal of ethyl acetate was confirmed by IR spectroscopy on the sublimed solid. The locations of the ethyl acetate stretching bands are consistent with those previously reported for metal-ethyl acetate complexes. ${ }^{2}$ 


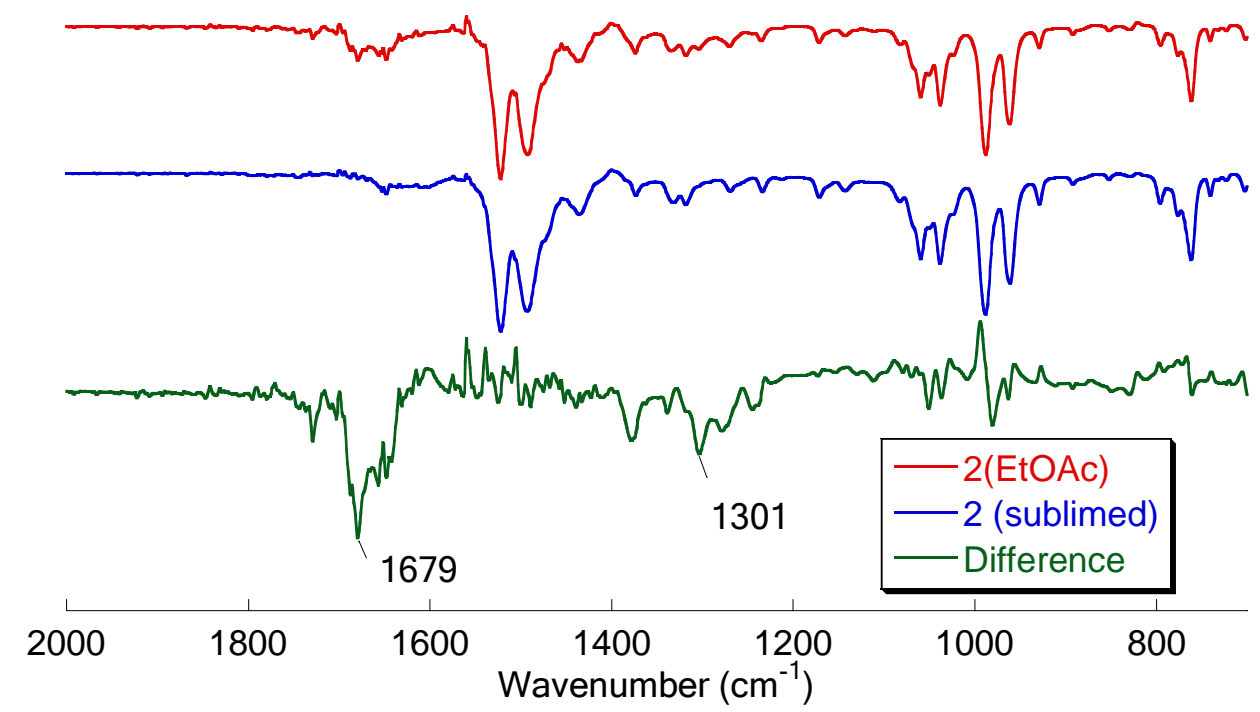

Figure S6. Removal of ethyl acetate from 2 by sublimation. $\mathrm{C}=\mathrm{O}$ vibrations in the realm of 1630-1700 $\mathrm{cm}^{-1}$ and $\mathrm{C}-\mathrm{O}^{\mathrm{Et}}$ bands near $1300 \mathrm{~cm}^{-1}$ present in the 2(EtOAc) EtOAc (red) disappear after sublimation (blue), and are more clearly apparent in the difference spectrum (green).

\section{Titration of 1 with EtOAc}

$3 \mathrm{~mL}$ of a $5.07 \times 10^{-5} \mathrm{M}$ solution of $\mathbf{1}$ was titrated with $54 \mu \mathrm{L}$ neat EtOAc in $1 \mu \mathrm{L}$ increments. The titration exhibited isosbestic behavior suggesting a simple two sided equilibrium between 1 and 1.EtOAc. The maximum absorbance change was found to be at $486 \mathrm{~nm}$, and the $\varepsilon$ value for the EtOAc adduct was found to be $2.46 \times 10^{4}$. With this and the known $\varepsilon$ value of $1.61 \mathrm{x}$ $10^{4}$ for $\mathbf{1}$, the relative concentrations of $\mathbf{1}, \mathbf{1} \cdot$ EtOAc, and EtOAc could be determined for each data point, and the equilibrium constant was determined by fitting the data to the equation $[\mathbf{1} \cdot \mathrm{EtOAc}]=\left(K_{E t O A c} C_{i}[\mathrm{EtOAc}]\right) /\left(1+K_{E t O A c}[\mathrm{EtOAc}]\right)$ to give $K_{E t O A c}=76 \pm 1$. 

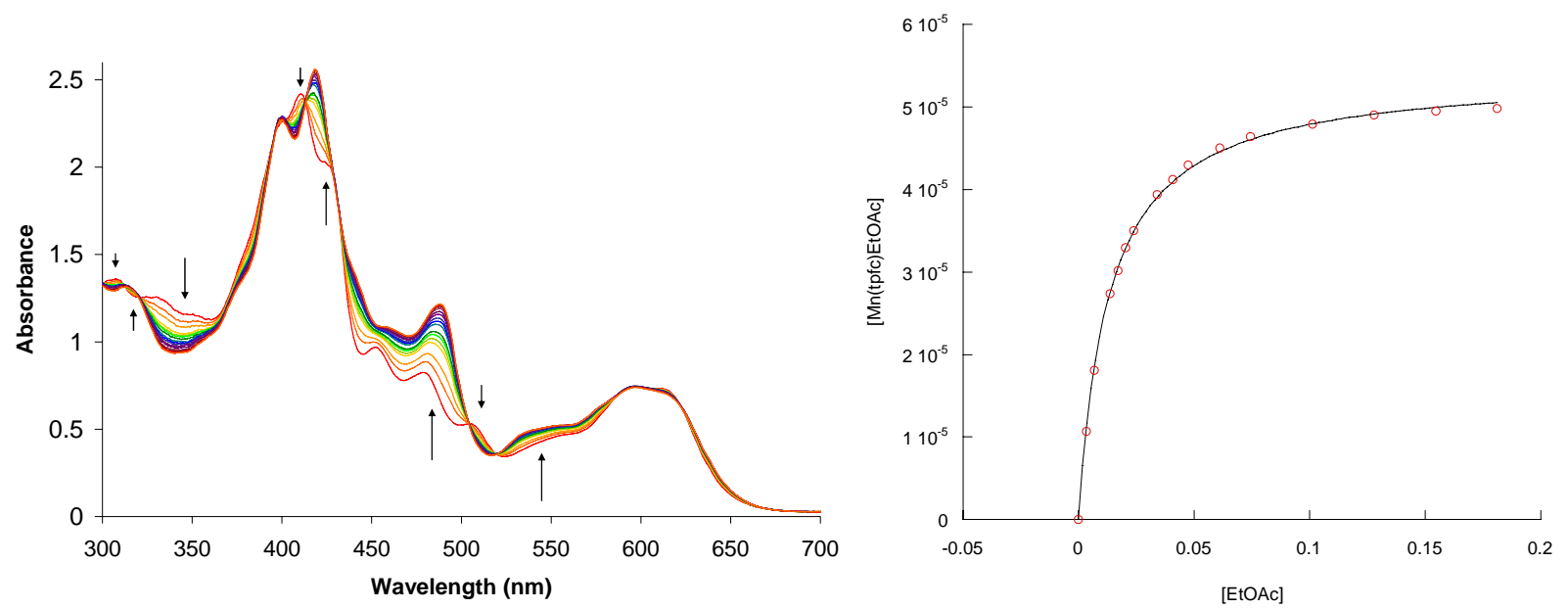

Figure S7. Left: Titration of 1 with EtOAc. The end spectrum represents an 3500-fold ethyl acetate excess. Right: Fit of $\mathbf{1} \cdot$ EtOAc concentration to the equation $[\mathbf{1} \cdot$ EtOAc] $=$ $\left(K_{\mathrm{eq}}[1]_{\mathrm{o}}[\mathrm{EtOAc}]\right) /\left(1+K_{\mathrm{eq}}[\right.$ EtOAc] $)$ (derivation in appendix). $K_{\mathrm{eq}}=76 \pm 1 . \mathrm{R}=0.9997$ for fit.

${ }^{1} \mathbf{H}$ NMR spectroscopy was carried out on (tpfc)MnO by stirring $\mathbf{1}(1.0 \mathrm{mg}, 0.98 \mu \mathrm{mol})$ with PhIO (2.0 mg, $9.1 \mu \mathrm{mol})$ in $\mathrm{CDCl}_{3}(5 \mathrm{~mL})$. The resulting red solution was analyzed by NMR. ${ }^{1} \mathrm{H}$ NMR ( $\mathrm{CD}_{2} \mathrm{Cl}_{2}$ ): $\delta 8.99$ (d, 2H, $\beta$-pyrrole), 9.03 (d, 2H, $\beta$-pyrrole), 9.24 (d, 2H, $\beta$-pyrrole), 9.56 (d, 2H, $\beta$-pyrrole).

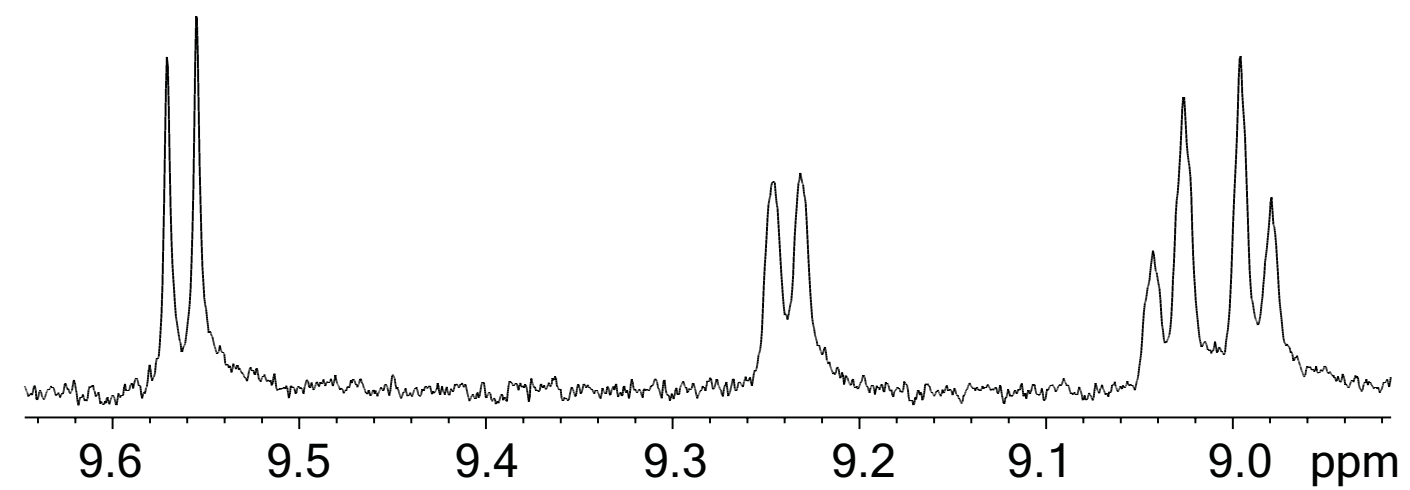

Figure S8. ${ }^{1} \mathrm{H}$ NMR spectrum of (tpfc) $\mathrm{Mn}=\mathrm{O}$ in $\mathrm{CD}_{2} \mathrm{Cl}_{2}$, generated in situ at $22{ }^{\circ} \mathrm{C}, 300 \mathrm{MHz}$. 


\section{Electronic Absorption Spectrum of (tpfc)Mn=0}

(tpfc)Mn=O was prepared in situ by the addition of 5 equivalents of solid PhIO to a $1 \times 10^{-5} \mathrm{M}$ solution of 1 in $\mathrm{CH}_{2} \mathrm{Cl}_{2}$. After stirring 10 min, the excess solid $\mathrm{PhIO}$ was removed by filtration, and the solution was analyzed by absorption spectroscopy.

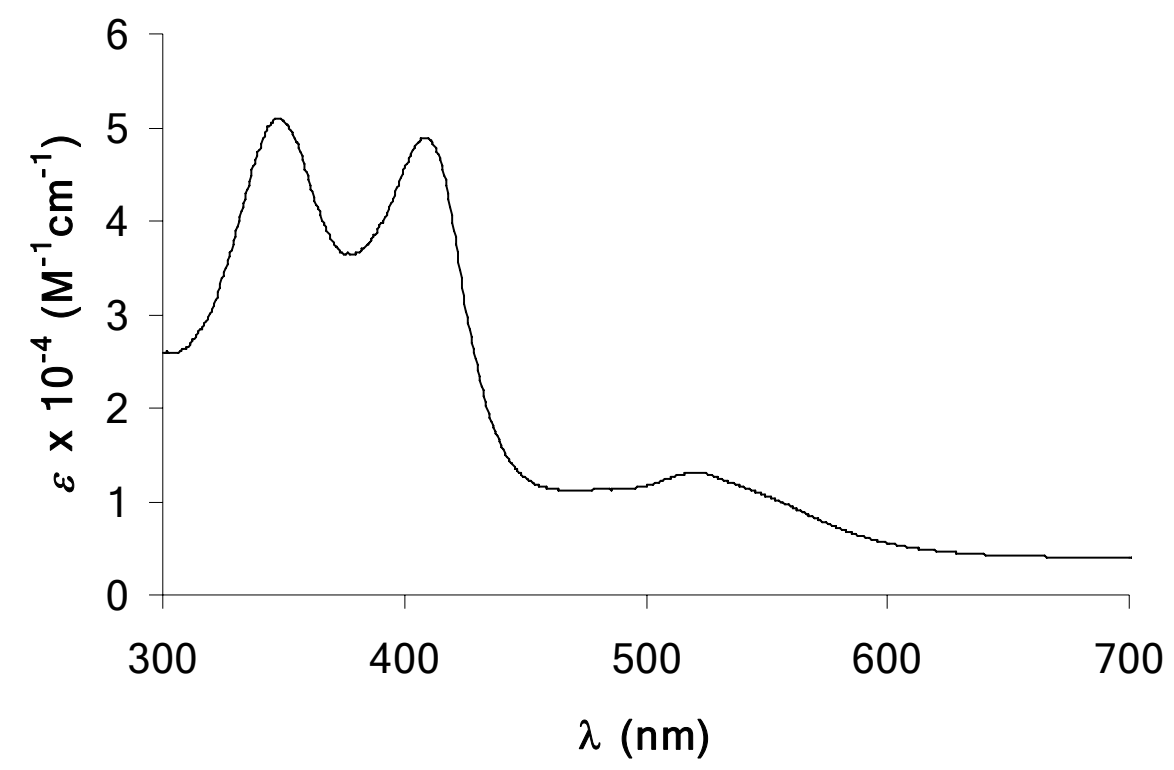

Figure S9. Absorption spectrum of (tpfc)Mn=O prepared in situ in $\mathrm{CH}_{2} \mathrm{Cl}_{2}, 22^{\circ} \mathrm{C}$.

\section{Magnetic Measurements by Evans method.}

The magnetic moment of complexes $\mathbf{1}, \mathbf{1}(\mathrm{py})_{2}$ (py = pyridine), and $\mathbf{2}$ were measured by Evans method. ${ }^{3} \mathbf{1}(\mathrm{py})_{2}$ was prepared by adding 100 equivalents of pyridine to a $2.15 \times 10^{-3} \mathrm{M}$ solution of $\mathbf{1}$ in bezene- $d_{6}$. For measurement, a solution of the complex (typically $1-5 \times 10^{-3} \mathrm{M}$ ) in benzene or toluene was placed inside a melting point capillary, which was placed into an NMR tube containing pure solvent of the same kind. This coaxial tube setup was analyzed in the spectrometer, and the magnetic moment was determined from the residual protio signal shift. $\mu_{\text {eff }}$ for, $\mathbf{1}(\mathrm{py})_{2}(\mathrm{py}=$ pyridine), and $\mathbf{2}$ were $4.72,5.28$, and 3.24 respectively. Ligation of $\mathbf{1}$ by pyridine was confirmed by absorption spectroscopy. 


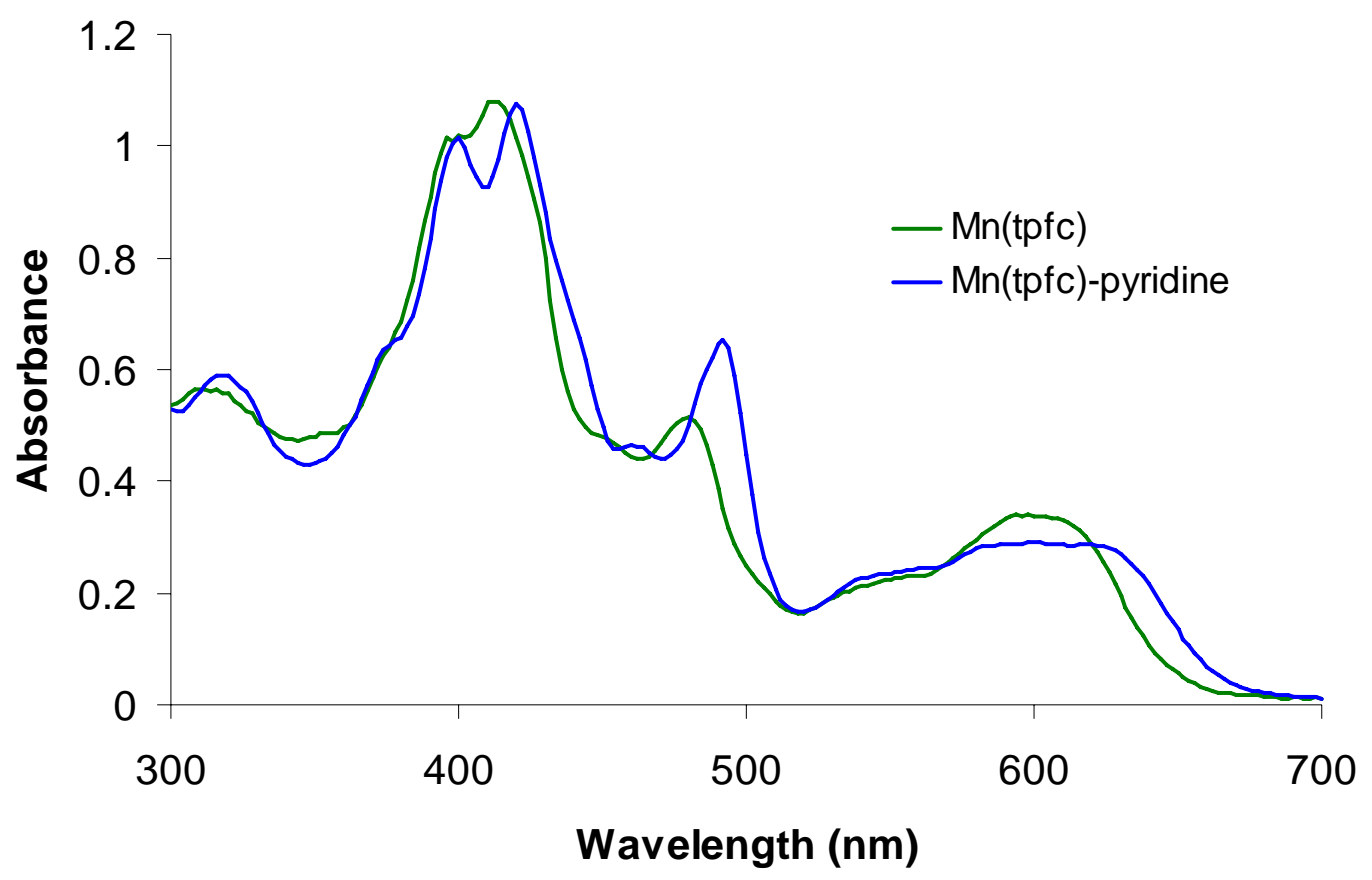

Figure S10. Electronic Absorption spectra of $\mathbf{1}$ and $\mathbf{1}$-pyridine adduct in benzene- $d_{6}$ (solutions described above) in a $0.1 \mathrm{~mm}$ pathlength cell. Addition of 100 eq. pyridine red-shifts the Qband signals, and changes the Soret to a resolved pair of bands. 


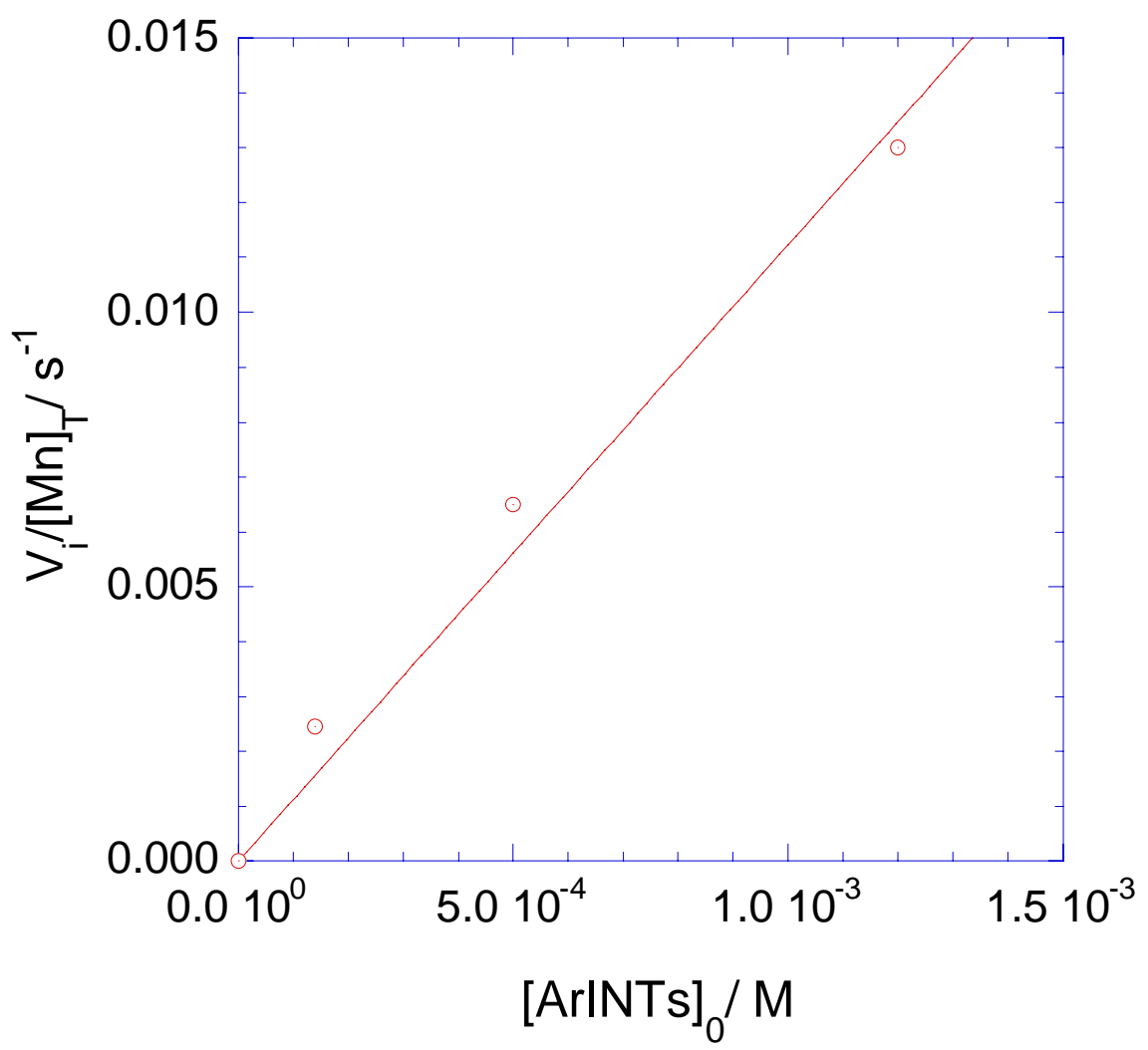

Figure S11. Initial rate versus [ArINTs] for catalytic aziridination of styrene. Slope $=11.2 \pm$ $0.6 \mathrm{~L} \mathrm{~mol}^{-1} \mathrm{~s}^{-1}\left(\mathrm{R}^{2}=0.981\right)$. 


\section{Crystal Data.}

Table S1.1. CRYSTAL DATA AND DATA COLLECTION PARAMETERS FOR

\section{$\mathrm{Mn}(\mathrm{tpfc})$ EtOAc·EtOAc (1)}

formula

formula weight

space group

a, $\AA$

b, $\AA$

c, $\AA$

$\beta$, deg

$\mathrm{V}, \AA^{3}$

$\mathrm{Z}$

$\mathrm{d}_{\text {calc }}, \mathrm{g} \mathrm{cm}^{-3}$

crystal dimensions, $\mathrm{mm}$

temperature, $\mathrm{K}$

radiation (wavelength, $\AA$ )

monochromator

linear abs coef, $\mathrm{mm}^{-1}$

absorption correction applied

transmission factors: min, $\max$

diffractometer

$\mathrm{h}, \mathrm{k}$, l range

$2 \theta$ range, deg

mosaicity, deg

programs used

$\mathrm{F}_{000}$

weighting

$1 /\left[\sigma^{2}\left(\mathrm{Fo}^{2}\right)+(0.0071 \mathrm{P})^{2}+15.3405 \mathrm{P}\right]$ where $\mathrm{P}=\left(\mathrm{Fo}^{2}+2 \mathrm{Fc}^{2}\right) / 3$

data collected

unique data

$\mathrm{R}_{\mathrm{int}}$

data used in refinement

cutoff used in R-factor calculations

data with $\mathrm{I}>2.0 \sigma(\mathrm{I})$

number of variables

largest shift/esd in final cycle

$\mathrm{R}\left(\mathrm{F}_{\mathrm{o}}\right)$

$\mathrm{R}_{\mathrm{w}}\left(\mathrm{F}_{\mathrm{o}}^{2}\right)$

goodness of fit

absolute structure determination
$\mathrm{C}_{45} \mathrm{H}_{24} \mathrm{~F}_{15} \mathrm{MnN}_{4} \mathrm{O}_{4}$ 1024.63

Cc (No. 9)

14.8543(4)

38.0136(15)

7.4550(2)

105.103(2)

4064.2(2)

4

1.674

$0.50 \times 0.13 \times 0.05$

150.

Mo $\mathrm{K}_{\alpha}(0.71073)$

graphite

0.428

empirical $^{\mathrm{a}}$

0.94, 0.98

Nonius KappaCCD

-19 to $19-49$ to 45 -9 to 9

4.29-54.97

0.57

SHELXTL

2056.0

21357

6321

0.273

6321

$\mathrm{F}_{0}^{2}>2.0 \sigma\left(\mathrm{F}_{0}^{2}\right)$

5724

627

0.00

0.060

0.105

1.127

Flack parameter ${ }^{\mathrm{b}}$ ( 0.47( 2))

a Otwinowski Z. \& Minor, W. Methods Enzymol. 1996,276307.

black, H. D. Acta Cryst., Sect. A 1983, A39, 876. 
Table S1.2. Table of Bond Distances in Angstroms

for $\mathrm{Mn}(\mathrm{tpfc}) \mathrm{EtOAc} \cdot \mathrm{EtOAc}(\mathbf{1})$

\begin{tabular}{|c|c|c|c|c|c|}
\hline Atom 1 & Atom 2 & Distance & Atom 1 & Atom 2 & Distance \\
\hline $\mathrm{Mn}$ & N1 & $1.900(4)$ & C9 & C10 & $1.408(7)$ \\
\hline Mn & N11 & $1.903(4)$ & C10 & C11 & $1.411(7)$ \\
\hline Mn & N16 & $1.913(4)$ & C10 & C101 & $1.485(7)$ \\
\hline Mn & N6 & $1.927(4)$ & C11 & C12 & $1.439(8)$ \\
\hline Mn & $\mathrm{O} 22$ & $2.172(4)$ & C12 & C13 & $1.363(8)$ \\
\hline F10 & C104 & $1.345(6)$ & C12 & H12 & $0.950(8)$ \\
\hline F52 & C52 & $1.337(6)$ & C13 & C14 & $1.418(8)$ \\
\hline F53 & C53 & $1.351(6)$ & C13 & H13 & $0.950(8)$ \\
\hline F54 & C54 & $1.346(6)$ & C14 & C15 & $1.398(8)$ \\
\hline F55 & C55 & $1.358(6)$ & C15 & C16 & $1.406(8)$ \\
\hline F56 & C56 & $1.350(6)$ & C15 & C151 & $1.512(7)$ \\
\hline F102 & C102 & $1.363(7)$ & C16 & C17 & $1.436(8)$ \\
\hline F103 & C103 & $1.344(7)$ & C17 & C18 & 1.393(8) \\
\hline F105 & C105 & $1.359(6)$ & C17 & H17 & $0.950(8)$ \\
\hline F106 & C106 & $1.345(6)$ & C18 & C19 & $1.403(8)$ \\
\hline F152 & C152 & $1.334(6)$ & C18 & H18 & $0.950(8)$ \\
\hline F153 & C153 & $1.335(6)$ & C21 & C22 & 1.461(9) \\
\hline F154 & C154 & $1.340(6)$ & C21 & H21A & 0.980(9) \\
\hline F155 & C155 & $1.337(7)$ & C21 & H21B & 0.980(9) \\
\hline F156 & C156 & $1.330(7)$ & C21 & H21C & 0.980(9) \\
\hline $\mathrm{O} 22$ & C22 & $1.204(7)$ & C24 & C25 & $1.395(11)$ \\
\hline $\mathrm{O} 23$ & C22 & $1.317(6)$ & C24 & H24A & $0.990(11)$ \\
\hline $\mathrm{O} 23$ & C24 & $1.584(9)$ & C24 & H24B & $0.990(11)$ \\
\hline O92 & C92 & 1.196(9) & C25 & H25A & $0.980(11)$ \\
\hline O93 & C92 & $1.336(8)$ & C25 & H25B & $0.980(11)$ \\
\hline O93 & C94 & $1.438(8)$ & C25 & H25C & $0.980(11)$ \\
\hline N1 & $\mathrm{C} 4$ & $1.370(6)$ & C51 & C56 & $1.370(7)$ \\
\hline N1 & C1 & $1.370(7)$ & C51 & C52 & 1.381(7) \\
\hline N6 & C9 & $1.367(7)$ & C52 & C53 & $1.386(7)$ \\
\hline N6 & C6 & $1.398(7)$ & C53 & C54 & $1.375(8)$ \\
\hline N11 & C11 & $1.383(7)$ & C54 & C55 & $1.346(8)$ \\
\hline N11 & C14 & $1.402(6)$ & C55 & C56 & $1.386(7)$ \\
\hline N16 & C16 & $1.352(6)$ & C91 & C92 & $1.523(10)$ \\
\hline N16 & C19 & $1.381(7)$ & C91 & H91A & 0.980(10) \\
\hline C1 & $\mathrm{C} 2$ & $1.410(8)$ & C91 & H91B & $0.980(10)$ \\
\hline C1 & C19 & $1.437(7)$ & C91 & H91C & $0.980(10)$ \\
\hline $\mathrm{C} 2$ & C3 & $1.379(8)$ & C94 & C95 & $1.504(10)$ \\
\hline C2 & $\mathrm{H} 2$ & $0.950(8)$ & C94 & H94A & 0.990(10) \\
\hline C3 & $\mathrm{C} 4$ & $1.426(8)$ & C94 & H94B & 0.990(10) \\
\hline C3 & H3 & $0.950(8)$ & C95 & H95A & $0.980(10)$ \\
\hline $\mathrm{C} 4$ & C5 & $1.409(7)$ & C95 & H95B & $0.980(10)$ \\
\hline C5 & C6 & $1.390(7)$ & C95 & H95C & $0.980(10)$ \\
\hline C5 & C51 & $1.508(7)$ & C101 & C102 & $1.370(8)$ \\
\hline C6 & C7 & $1.422(7)$ & C101 & C106 & 1.394(8) \\
\hline $\mathrm{C} 7$ & C8 & $1.372(8)$ & C102 & C103 & $1.363(9)$ \\
\hline $\mathrm{C} 7$ & H7 & $0.950(8)$ & C103 & C104 & $1.377(9)$ \\
\hline C8 & C9 & $1.435(7)$ & C104 & C105 & $1.360(8)$ \\
\hline C8 & H8 & $0.950(7)$ & C105 & C106 & $1.374(8)$ \\
\hline
\end{tabular}




\section{Bond Distances (cont.)}

\begin{tabular}{|c|c|c|c|c|c|}
\hline$\underline{\text { Atom }} \underline{1}$ & Atom 2 & Distance & Atom 1 & Atom 2 & Distance \\
\hline$\overline{\mathrm{C} 151}$ & $\overline{C 152}$ & $1.366(8)$ & $\overline{\mathrm{C} 153}$ & C154 & $1.379(8)$ \\
\hline C151 & C156 & $1.379(8)$ & C154 & C155 & $1.374(8)$ \\
\hline C152 & C153 & $1.400(7)$ & C155 & C156 & $1.381(8)$ \\
\hline
\end{tabular}

Numbers in parentheses are estimated standard deviations in the least significant digits. 
Table S1.3. Table of Bond Angles in Degrees

for Mn(tpfc)EtOAc·EtOAc (1)

\begin{tabular}{|c|c|c|c|c|c|c|c|}
\hline Atom 1 & Atom $\underline{2}$ & $\underline{\text { Atom }} \underline{3}$ & $\underline{\text { Angle }}$ & $\underline{\text { Atom }} 1$ & $\underline{\text { Atom }} \underline{2}$ & $\underline{\text { Atom }} \underline{3}$ & $\underline{\text { Angle }}$ \\
\hline$\overline{N 1}$ & $\overline{\mathrm{Mn}}$ & $\overline{N 11}$ & $1 \overline{64.43(18)}$ & $\overline{\mathrm{C} 9}$ & $\overline{\mathrm{C} 8}$ & $\overline{\mathrm{H} 8}$ & $1 \overline{26.5(5)}$ \\
\hline N1 & Mn & N16 & 80.73(18) & N6 & C9 & C10 & $124.6(5)$ \\
\hline N11 & Mn & N16 & 89.88(18) & N6 & C9 & C8 & 108.3(5) \\
\hline N1 & Mn & N6 & $90.42(18)$ & C10 & C9 & C8 & $127.0(5)$ \\
\hline N11 & Mn & N6 & $96.02(19)$ & C9 & C10 & C11 & $124.9(5)$ \\
\hline N16 & Mn & N6 & $165.35(18)$ & C9 & C10 & C101 & $118.6(5)$ \\
\hline N1 & Mn & $\mathrm{O} 22$ & $97.87(17)$ & C11 & C10 & C101 & $116.5(5)$ \\
\hline N11 & Mn & $\mathrm{O} 22$ & $96.11(17)$ & N11 & C11 & C10 & $124.9(5)$ \\
\hline N16 & $\mathrm{Mn}$ & $\mathrm{O} 22$ & 101.11(17) & N11 & C11 & C12 & $108.4(5)$ \\
\hline N6 & $\mathrm{Mn}$ & $\mathrm{O} 22$ & $91.60(17)$ & C10 & C11 & C12 & $126.7(5)$ \\
\hline C22 & $\mathrm{O} 22$ & $\mathrm{Mn}$ & $155.3(4)$ & C13 & C12 & C11 & $107.0(5)$ \\
\hline $\mathrm{C} 22$ & $\mathrm{O} 23$ & C24 & $116.4(5)$ & $\mathrm{C} 13$ & C12 & H12 & $126.5(5)$ \\
\hline C92 & O93 & C94 & $116.7(6)$ & C11 & C12 & H12 & $126.5(5)$ \\
\hline C4 & N1 & C1 & 109.7(4) & C12 & C13 & C14 & 109.3(5) \\
\hline C4 & N1 & Mn & $132.5(4)$ & C12 & C13 & H13 & 125.3(5) \\
\hline C1 & N1 & $\mathrm{Mn}$ & 117.8(3) & C14 & C13 & H13 & $125.3(5)$ \\
\hline C9 & N6 & C6 & $108.8(4)$ & C15 & C14 & N11 & $124.0(5)$ \\
\hline C9 & N6 & Mn & 124.5(3) & C15 & C14 & C13 & 128.5(5) \\
\hline C6 & N6 & Mn & $126.5(4)$ & N11 & C14 & C13 & 107.4(5) \\
\hline C11 & N11 & C14 & $107.9(4)$ & C14 & C15 & C16 & $124.4(5)$ \\
\hline C11 & N11 & Mn & 124.2(3) & C14 & C15 & C151 & $115.7(5)$ \\
\hline C14 & N11 & $\mathrm{Mn}$ & $127.6(4)$ & C16 & C15 & C151 & $119.8(5)$ \\
\hline C16 & N16 & C19 & $110.0(4)$ & N16 & C16 & C15 & $120.4(5)$ \\
\hline C16 & N16 & $\mathrm{Mn}$ & $132.7(4)$ & N16 & C16 & C17 & 106.9(5) \\
\hline C19 & N16 & Mn & 117.4(3) & C15 & C16 & C17 & 132.3(5) \\
\hline N1 & C1 & C2 & 108.2(5) & C18 & C17 & C16 & $107.9(5)$ \\
\hline N1 & C1 & C19 & $112.5(5)$ & C18 & C17 & H17 & $126.0(5)$ \\
\hline $\mathrm{C} 2$ & $\mathrm{C} 1$ & C19 & $139.4(5)$ & C16 & C17 & H17 & $126.0(5)$ \\
\hline C3 & C2 & C1 & $107.2(5)$ & C17 & C18 & C19 & $106.7(5)$ \\
\hline C3 & $\mathrm{C} 2$ & $\mathrm{H} 2$ & $126.4(5)$ & C17 & C18 & H18 & $126.6(5)$ \\
\hline C1 & C2 & $\mathrm{H} 2$ & $126.4(5)$ & C19 & C18 & H18 & 126.6(5) \\
\hline $\mathrm{C} 2$ & C3 & $\mathrm{C} 4$ & $108.2(5)$ & N16 & C19 & C18 & 108.5(5) \\
\hline C2 & C3 & H3 & 125.9(5) & N16 & C19 & C1 & $111.6(4)$ \\
\hline C4 & C3 & H3 & $125.9(5)$ & C18 & C19 & C1 & $139.8(5)$ \\
\hline N1 & C4 & C5 & $119.9(5)$ & $\mathrm{C} 22$ & C21 & $\mathrm{H} 21 \mathrm{~A}$ & 109.5(5) \\
\hline N1 & C4 & C3 & $106.8(4)$ & C22 & C21 & H21B & 109.5(5) \\
\hline C5 & C4 & C3 & 133.1(5) & H21A & C21 & H21B & 109.5(5) \\
\hline C6 & C5 & C4 & $125.0(5)$ & $\mathrm{C} 22$ & C21 & $\mathrm{H} 21 \mathrm{C}$ & 109.5(5) \\
\hline C6 & C5 & C51 & $117.6(5)$ & H21A & C21 & H21C & 109.5(5) \\
\hline C4 & C5 & C51 & $117.4(5)$ & H21B & C21 & H21C & 109.5(5) \\
\hline C5 & C6 & N6 & $124.6(5)$ & $\mathrm{O} 22$ & C22 & $\mathrm{O} 23$ & 121.6(5) \\
\hline C5 & C6 & C7 & $128.3(5)$ & $\mathrm{O} 22$ & C22 & C21 & $125.7(6)$ \\
\hline N6 & C6 & C7 & 107.1(5) & $\mathrm{O} 23$ & C22 & C21 & $112.5(5)$ \\
\hline C8 & C7 & C6 & 108.7(4) & C25 & C24 & $\mathrm{O} 23$ & 101.6(7) \\
\hline C8 & C7 & H7 & $125.6(4)$ & C25 & C24 & H24A & $111.5(7)$ \\
\hline C6 & C7 & H7 & $125.6(4)$ & $\mathrm{O} 23$ & C24 & H24A & $111.5(7)$ \\
\hline C7 & C8 & C9 & 107.1(5) & $\mathrm{C} 25$ & C24 & H24B & 111.5(7) \\
\hline C7 & C8 & H8 & $126.5(5)$ & $\mathrm{O} 23$ & C24 & H24B & 111.5(7) \\
\hline
\end{tabular}


Bond Angles (cont.)

\begin{tabular}{|c|c|c|c|c|c|c|c|}
\hline Atom 1 & $\underline{\text { Atom }} \underline{2}$ & Atom $\underline{3}$ & Angle & $\underline{\text { Atom }} 1$ & $\underline{\text { Atom }} \underline{2}$ & $\underline{\text { Atom }} \underline{3}$ & $\underline{\text { Angle }}$ \\
\hline$\overline{\mathrm{H} 24 \mathrm{~A}}$ & $\overline{\mathrm{C} 24}$ & $\overline{\mathrm{H}} 24 \mathrm{~B}$ & $109.3(7)$ & $\overline{\mathrm{C} 94}$ & $\overline{\mathrm{C} 95}$ & $\overline{\mathrm{H} 95 \mathrm{~B}}^{-}$ & $109.5(6)$ \\
\hline C24 & C25 & H25A & $109.5(7)$ & H95A & C95 & Н95B & $109.5(6)$ \\
\hline C24 & $\mathrm{C} 25$ & H25B & 109.5(7) & C94 & C95 & H95C & 109.5(6) \\
\hline $\mathrm{H} 25 \mathrm{~A}$ & $\mathrm{C} 25$ & H25B & 109.5(7) & H95A & C95 & H95C & 109.5(6) \\
\hline C24 & C25 & H25C & $109.5(7)$ & H95B & C95 & H95C & 109.5(6) \\
\hline H25A & C25 & H25C & 109.5(7) & C102 & C101 & C106 & $115.2(5)$ \\
\hline H25B & C25 & $\mathrm{H} 25 \mathrm{C}$ & 109.5(7) & C102 & C101 & C10 & $123.4(5)$ \\
\hline C56 & C51 & C52 & 116.4(5) & C106 & C101 & C10 & 121.3(5) \\
\hline C56 & C51 & C5 & $122.6(5)$ & F102 & C102 & C103 & $117.2(5)$ \\
\hline C52 & C51 & C5 & $121.0(5)$ & F102 & C102 & C101 & $118.4(5)$ \\
\hline F52 & C52 & C51 & $120.8(5)$ & C103 & C102 & C101 & $124.4(6)$ \\
\hline F52 & C52 & C53 & $117.3(5)$ & F103 & C103 & C102 & $121.7(6)$ \\
\hline C51 & C52 & C53 & $121.9(5)$ & F103 & C103 & C104 & $119.9(6)$ \\
\hline F53 & C53 & C54 & $119.9(5)$ & C102 & C103 & C104 & 118.3(5) \\
\hline F53 & C53 & C52 & $120.2(5)$ & F10 & C104 & C105 & $120.0(6)$ \\
\hline C54 & C53 & C52 & $119.8(5)$ & F10 & C104 & C103 & $120.0(6)$ \\
\hline F54 & C54 & C55 & $121.5(5)$ & C105 & C104 & C103 & $120.0(5)$ \\
\hline F54 & C54 & C53 & $119.3(5)$ & F105 & C105 & C104 & $119.8(5)$ \\
\hline C55 & C54 & C53 & 119.2(5) & F105 & C105 & C106 & $120.0(5)$ \\
\hline C54 & C55 & F55 & $119.7(5)$ & C104 & C105 & C106 & $120.1(5)$ \\
\hline C54 & C55 & C56 & $120.6(5)$ & F106 & C106 & C105 & $118.7(5)$ \\
\hline F55 & C55 & C56 & $119.6(5)$ & F106 & C106 & C101 & 119.5(5) \\
\hline F56 & C56 & C51 & 120.1(5) & C105 & C106 & C101 & 121.8(5) \\
\hline F56 & C56 & C55 & $117.9(5)$ & C152 & C151 & C156 & $117.9(5)$ \\
\hline C51 & C56 & C55 & 122.1(5) & C152 & C151 & C15 & 121.5(5) \\
\hline C92 & C91 & H91A & 109.5(5) & C156 & C151 & C15 & $120.5(5)$ \\
\hline C92 & C91 & H91B & 109.5(5) & F152 & C152 & C151 & $120.9(5)$ \\
\hline H91A & C91 & H91B & 109.5(5) & F152 & C152 & C153 & $117.4(5)$ \\
\hline C92 & C91 & H91C & 109.5(5) & C151 & C152 & C153 & $121.6(5)$ \\
\hline H91A & C91 & H91C & 109.5(5) & F153 & C153 & C154 & $120.2(5)$ \\
\hline H91B & C91 & H91C & 109.5(5) & F153 & C153 & C152 & $120.9(5)$ \\
\hline O92 & C92 & O93 & $123.0(7)$ & C154 & C153 & C152 & $118.9(5)$ \\
\hline O92 & C92 & C91 & $125.8(7)$ & F154 & C154 & C155 & 120.5(5) \\
\hline O93 & C92 & C91 & $111.2(6)$ & F154 & C154 & C153 & 119.1(5) \\
\hline O93 & C94 & C95 & 106.9(6) & C155 & C154 & C153 & $120.3(5)$ \\
\hline O93 & C94 & H94A & $110.3(6)$ & F155 & C155 & C154 & 119.3(5) \\
\hline C95 & C94 & H94A & $110.3(6)$ & F155 & C155 & C156 & $121.4(5)$ \\
\hline O93 & C94 & H94B & $110.3(6)$ & C154 & C155 & C156 & 119.3(5) \\
\hline C95 & C94 & H94B & $110.3(6)$ & F156 & C156 & C151 & $121.0(5)$ \\
\hline H94A & C94 & H94B & $108.6(6)$ & F156 & C156 & C155 & 117.1(5) \\
\hline C94 & C95 & H95A & 109.5(6) & C151 & C156 & C155 & 121.9(5) \\
\hline
\end{tabular}

Numbers in parentheses are estimated standard deviations in the least significant digits. 
Table S1.4. Table of Torsional Angles in Degrees

\section{for Mn(tpfc)EtOAc·EtOAc (1)}

\begin{tabular}{|c|c|c|c|c|}
\hline Atom 1 & Atom 2 & Atom $\underline{3}$ & Atom $\underline{4}$ & Angle \\
\hline $\mathrm{N}(1)$ & $\mathrm{Mn}$ & $\mathrm{O}(22)$ & $\mathrm{C}(22)$ & $-144.72(1.01)$ \\
\hline $\mathrm{N}(6)$ & $\mathrm{Mn}$ & $\mathrm{O}(22)$ & $\mathrm{C}(22)$ & $124.64(1.02)$ \\
\hline $\mathrm{N}(11)$ & Mn & $\mathrm{O}(22)$ & $\mathrm{C}(22)$ & $28.40(1.03)$ \\
\hline $\mathrm{N}(16)$ & Mn & $\mathrm{O}(22)$ & $\mathrm{C}(22)$ & $-62.67(1.03)$ \\
\hline $\mathrm{O}(22)$ & $\mathrm{Mn}$ & $\mathrm{N}(1)$ & $\mathrm{C}(1)$ & 99.51 ( 0.38$)$ \\
\hline $\mathrm{O}(22)$ & $\mathrm{Mn}$ & $\mathrm{N}(1)$ & $\mathrm{C}(4)$ & $-82.77(0.48)$ \\
\hline $\mathrm{N}(6)$ & $\mathrm{Mn}$ & $\mathrm{N}(1)$ & $\mathrm{C}(1)$ & $-168.80(0.39)$ \\
\hline $\mathrm{N}(6)$ & Mn & $\mathrm{N}(1)$ & $\mathrm{C}(4)$ & $8.93(0.49)$ \\
\hline $\mathrm{N}(11)$ & $\mathrm{Mn}$ & $\mathrm{N}(1)$ & $\mathrm{C}(1)$ & $-54.14(0.89)$ \\
\hline $\mathrm{N}(11)$ & $\mathrm{Mn}$ & $\mathrm{N}(1)$ & $\mathrm{C}(4)$ & $123.58(0.73)$ \\
\hline $\mathrm{N}(16)$ & $\mathrm{Mn}$ & $\mathrm{N}(1)$ & $\mathrm{C}(1)$ & $-0.54(0.37)$ \\
\hline $\mathrm{N}(16)$ & Mn & $\mathrm{N}(1)$ & $\mathrm{C}(4)$ & $177.19(0.50)$ \\
\hline $\mathrm{O}(22)$ & $\mathrm{Mn}$ & $\mathrm{N}(6)$ & $\mathrm{C}(6)$ & $87.66(0.42)$ \\
\hline $\mathrm{O}(22)$ & $\mathrm{Mn}$ & $\mathrm{N}(6)$ & $\mathrm{C}(9)$ & $-87.83(0.42)$ \\
\hline $\mathrm{N}(1)$ & $\mathrm{Mn}$ & $\mathrm{N}(6)$ & $\mathrm{C}(6)$ & $-10.21(0.43)$ \\
\hline $\mathrm{N}(1)$ & $\mathrm{Mn}$ & $\mathrm{N}(6)$ & $\mathrm{C}(9)$ & $174.29(0.42)$ \\
\hline $\mathrm{N}(11)$ & $\mathrm{Mn}$ & $\mathrm{N}(6)$ & $\mathrm{C}(6)$ & $-176.02(0.42)$ \\
\hline $\mathrm{N}(11)$ & $\mathrm{Mn}$ & $\mathrm{N}(6)$ & $\mathrm{C}(9)$ & $8.49(0.43)$ \\
\hline $\mathrm{N}(16)$ & Mn & $\mathrm{N}(6)$ & $\mathrm{C}(6)$ & $-62.76(0.94)$ \\
\hline $\mathrm{N}(16)$ & $\mathrm{Mn}$ & $\mathrm{N}(6)$ & $\mathrm{C}(9)$ & $121.75(0.74)$ \\
\hline $\mathrm{O}(22)$ & Mn & $\mathrm{N}(11)$ & $\mathrm{C}(11)$ & 83.57 ( 0.42) \\
\hline $\mathrm{O}(22)$ & $\mathrm{Mn}$ & $\mathrm{N}(11)$ & $\mathrm{C}(14)$ & $-90.45(0.43)$ \\
\hline $\mathrm{N}(1)$ & $\mathrm{Mn}$ & $\mathrm{N}(11)$ & $\mathrm{C}(11)$ & $-122.67(0.71)$ \\
\hline $\mathrm{N}(1)$ & Mn & $\mathrm{N}(11)$ & $\mathrm{C}(14)$ & $63.30(0.90)$ \\
\hline $\mathrm{N}(6)$ & $\mathrm{Mn}$ & $\mathrm{N}(11)$ & $\mathrm{C}(11)$ & $-8.72(0.43)$ \\
\hline $\mathrm{N}(6)$ & $\mathrm{Mn}$ & $\mathrm{N}(11)$ & $\mathrm{C}(14)$ & $177.25(0.43)$ \\
\hline $\mathrm{N}(16)$ & $\mathrm{Mn}$ & $\mathrm{N}(11)$ & $\mathrm{C}(11)$ & $-175.28(0.42)$ \\
\hline $\mathrm{N}(16)$ & Mn & $\mathrm{N}(11)$ & $\mathrm{C}(14)$ & $10.70(0.44)$ \\
\hline $\mathrm{O}(22)$ & $\mathrm{Mn}$ & $\mathrm{N}(16)$ & $\mathrm{C}(16)$ & 86.77 ( 0.51$)$ \\
\hline $\mathrm{O}(22)$ & $\mathrm{Mn}$ & $\mathrm{N}(16)$ & C(19) & $-95.19(0.38)$ \\
\hline $\mathrm{N}(1)$ & $\mathrm{Mn}$ & $\mathrm{N}(16)$ & $\mathrm{C}(16)$ & $-176.96(0.53)$ \\
\hline $\mathrm{N}(1)$ & $\mathrm{Mn}$ & $\mathrm{N}(16)$ & $\mathrm{C}(19)$ & $1.09(0.37)$ \\
\hline $\mathrm{N}(6)$ & $\mathrm{Mn}$ & N(16) & $\mathrm{C}(16)$ & $-123.42(0.78)$ \\
\hline $\mathrm{N}(6)$ & $\mathrm{Mn}$ & $\mathrm{N}(16)$ & C(19) & $54.62(0.92)$ \\
\hline $\mathrm{N}(11)$ & $\mathrm{Mn}$ & N(16) & $\mathrm{C}(16)$ & $-9.43(0.51)$ \\
\hline $\mathrm{N}(11)$ & $\mathrm{Mn}$ & N(16) & $\mathrm{C}(19)$ & 168.61 ( 0.38) \\
\hline Mn & $\mathrm{O}(22)$ & $\mathrm{C}(22)$ & $\mathrm{O}(23)$ & $-150.89(0.77)$ \\
\hline $\mathrm{Mn}$ & $\mathrm{O}(22)$ & $\mathrm{C}(22)$ & $\mathrm{C}(21)$ & $35.72(1.41)$ \\
\hline $\mathrm{C}(24)$ & $\mathrm{O}(23)$ & $\mathrm{C}(22)$ & $\mathrm{O}(22)$ & $-0.30(0.96)$ \\
\hline $\mathrm{C}(24)$ & $\mathrm{O}(23)$ & $\mathrm{C}(22)$ & $\mathrm{C}(21)$ & 173.89 ( 0.59$)$ \\
\hline $\mathrm{C}(22)$ & $\mathrm{O}(23)$ & $\mathrm{C}(24)$ & $C(25)$ & $92.33(0.79)$ \\
\hline C(94) & $\mathrm{O}(93)$ & $\mathrm{C}(92)$ & $\mathrm{O}(92)$ & $-2.19(1.30)$ \\
\hline $\mathrm{C}(94)$ & $\mathrm{O}(93)$ & $\mathrm{C}(92)$ & $\mathrm{C}(91)$ & $177.93(0.70)$ \\
\hline $\mathrm{C}(92)$ & $\mathrm{O}(93)$ & $\mathrm{C}(94)$ & C(95) & $177.36(0.72)$ \\
\hline Mn & $\mathrm{N}(1)$ & $\mathrm{C}(1)$ & $\mathrm{C}(2)$ & $179.79(0.35)$ \\
\hline
\end{tabular}


Table of Torsional Angles in Degrees (cont)

for $\mathrm{Mn}(\mathrm{tpfc}) \mathrm{EtOAc} \cdot \mathrm{EtOAc}(\mathbf{1})$

\begin{tabular}{|c|c|c|c|c|}
\hline$\underline{\text { Atom }} \underline{1}$ & $\underline{\text { Atom }} \underline{2}$ & $\underline{\text { Atom }} \underline{3}$ & Atom 4 & Angle \\
\hline $\mathrm{Mn}$ & $\mathrm{N}(1)$ & $\mathrm{C}(1)$ & $\mathrm{C}(19)$ & -0.07 ( 0.59) \\
\hline $\mathrm{C}(4)$ & $\mathrm{N}(1)$ & $\mathrm{C}(1)$ & $C(2)$ & 1.57 ( 0.59) \\
\hline C(4) & $\mathrm{N}(1)$ & $C(1)$ & $\mathrm{C}(19)$ & $-178.29(0.43)$ \\
\hline $\mathrm{Mn}$ & $\mathrm{N}(1)$ & $\mathrm{C}(4)$ & $\mathrm{C}(3)$ & -179.58 ( 0.37) \\
\hline Mn & $\mathrm{N}(1)$ & $\mathrm{C}(4)$ & $\mathrm{C}(5)$ & $-2.98(0.75)$ \\
\hline $\mathrm{C}(1)$ & $\mathrm{N}(1)$ & $\mathrm{C}(4)$ & $\mathrm{C}(3)$ & $-1.72(0.57)$ \\
\hline $\mathrm{C}(1)$ & $\mathrm{N}(1)$ & $C(4)$ & $C(5)$ & $174.88(0.46)$ \\
\hline $\mathrm{Mn}$ & $\mathrm{N}(6)$ & $C(6)$ & $C(5)$ & $6.61(0.73)$ \\
\hline $\mathrm{Mn}$ & $\mathrm{N}(6)$ & $\mathrm{C}(6)$ & $\mathrm{C}(7)$ & $-176.30(0.34)$ \\
\hline $\mathrm{C}(9)$ & $\mathrm{N}(6)$ & $C(6)$ & $C(5)$ & $-177.31(0.49)$ \\
\hline $\mathrm{C}(9)$ & $\mathrm{N}(6)$ & $C(6)$ & $C(7)$ & $-0.21(0.56)$ \\
\hline $\mathrm{Mn}$ & $\mathrm{N}(6)$ & $\mathrm{C}(9)$ & $\mathrm{C}(8)$ & $176.27(0.34)$ \\
\hline $\mathrm{Mn}$ & $\mathrm{N}(6)$ & $\mathrm{C}(9)$ & $\mathrm{C}(10)$ & $-4.20(0.72)$ \\
\hline$C(6)$ & $\mathrm{N}(6)$ & $\mathrm{C}(9)$ & $C(8)$ & $0.09(0.53)$ \\
\hline$C(6)$ & $\mathrm{N}(6)$ & $\mathrm{C}(9)$ & $\mathrm{C}(10)$ & $179.62(0.48)$ \\
\hline $\mathrm{Mn}$ & $\mathrm{N}(11)$ & $\mathrm{C}(11)$ & $\mathrm{C}(10)$ & $4.86(0.72)$ \\
\hline $\mathrm{Mn}$ & $\mathrm{N}(11)$ & $\mathrm{C}(11)$ & $\mathrm{C}(12)$ & $-174.28(0.36)$ \\
\hline$C(14)$ & $\mathrm{N}(11)$ & $\mathrm{C}(11)$ & $C(10)$ & $179.89(0.50)$ \\
\hline $\mathrm{C}(14)$ & $\mathrm{N}(11)$ & $\mathrm{C}(11)$ & $\mathrm{C}(12)$ & $0.75(0.57)$ \\
\hline $\mathrm{Mn}$ & $\mathrm{N}(11)$ & $\mathrm{C}(14)$ & $\mathrm{C}(13)$ & 174.66 ( 0.37) \\
\hline $\mathrm{Mn}$ & $\mathrm{N}(11)$ & $\mathrm{C}(14)$ & $\mathrm{C}(15)$ & $-8.56(0.73)$ \\
\hline $\mathrm{C}(11)$ & $\mathrm{N}(11)$ & $\mathrm{C}(14)$ & $\mathrm{C}(13)$ & $-0.15(0.57)$ \\
\hline $\mathrm{C}(11)$ & $\mathrm{N}(11)$ & $\mathrm{C}(14)$ & $\mathrm{C}(15)$ & $176.63(0.48)$ \\
\hline $\mathrm{Mn}$ & $\mathrm{N}(16)$ & $C(16)$ & $\mathrm{C}(15)$ & $4.88(0.82)$ \\
\hline $\mathrm{Mn}$ & $\mathrm{N}(16)$ & $C(16)$ & $\mathrm{C}(17)$ & $179.29(0.41)$ \\
\hline C(19) & $\mathrm{N}(16)$ & $C(16)$ & $\mathrm{C}(15)$ & $-173.27(0.49)$ \\
\hline C(19) & $\mathrm{N}(16)$ & $C(16)$ & $\mathrm{C}(17)$ & $1.14(0.62)$ \\
\hline $\mathrm{Mn}$ & $\mathrm{N}(16)$ & C(19) & $\mathrm{C}(1)$ & $-1.39(0.56)$ \\
\hline $\mathrm{Mn}$ & $\mathrm{N}(16)$ & $\mathrm{C}(19)$ & C(18) & $-178.93(0.35)$ \\
\hline $\mathrm{C}(16)$ & $\mathrm{N}(16)$ & $\mathrm{C}(19)$ & $\mathrm{C}(1)$ & $177.07(0.45)$ \\
\hline $\mathrm{C}(16)$ & $\mathrm{N}(16)$ & $\mathrm{C}(19)$ & $\mathrm{C}(18)$ & $-0.47(0.60)$ \\
\hline $\mathrm{N}(1)$ & $\mathrm{C}(1)$ & $\mathrm{C}(2)$ & $\mathrm{C}(3)$ & $-0.76(0.61)$ \\
\hline $\mathrm{C}(19)$ & $\mathrm{C}(1)$ & $\mathrm{C}(2)$ & $C(3)$ & $179.04(0.62)$ \\
\hline $\mathrm{N}(1)$ & $\mathrm{C}(1)$ & $\mathrm{C}(19)$ & $\mathrm{N}(16)$ & $0.91(0.62)$ \\
\hline $\mathrm{N}(1)$ & $\mathrm{C}(1)$ & $\mathrm{C}(19)$ & $\mathrm{C}(18)$ & $177.29(0.64)$ \\
\hline $\mathrm{C}(2)$ & $\mathrm{C}(1)$ & $\mathrm{C}(19)$ & $\mathrm{N}(16)$ & $-178.88(0.63)$ \\
\hline $\mathrm{C}(2)$ & $\mathrm{C}(1)$ & C(19) & C(18) & $-2.50(1.24)$ \\
\hline $\mathrm{C}(1)$ & $C(2)$ & $C(3)$ & $\mathrm{C}(4)$ & $-0.29(0.61)$ \\
\hline$C(2)$ & $\mathrm{C}(3)$ & $\mathrm{C}(4)$ & $\mathrm{N}(1)$ & $1.23(0.59)$ \\
\hline $\mathrm{C}(2)$ & $\mathrm{C}(3)$ & $\mathrm{C}(4)$ & $\mathrm{C}(5)$ & $-174.74(0.56)$ \\
\hline $\mathrm{N}(1)$ & $\mathrm{C}(4)$ & $\mathrm{C}(5)$ & $\mathrm{C}(6)$ & $-5.22(0.79)$ \\
\hline $\mathrm{N}(1)$ & $\mathrm{C}(4)$ & $C(5)$ & C(51) & 177.17 ( 0.44) \\
\hline $\mathrm{C}(3)$ & $\mathrm{C}(4)$ & $\mathrm{C}(5)$ & $\mathrm{C}(6)$ & $170.32(0.54)$ \\
\hline $\mathrm{C}(3)$ & $\mathrm{C}(4)$ & $\mathrm{C}(5)$ & C(51) & $-7.28(0.85)$ \\
\hline$C(4)$ & $\mathrm{C}(5)$ & $\mathrm{C}(6)$ & $\mathrm{N}(6)$ & $3.23(0.83)$ \\
\hline $\mathrm{C}(4)$ & $C(5)$ & $\mathrm{C}(6)$ & $\mathrm{C}(7)$ & $-173.23(0.51)$ \\
\hline
\end{tabular}


Table of Torsional Angles in Degrees (cont)

for $\mathrm{Mn}(\mathrm{tpfc}) \mathrm{EtOAc} \cdot \mathrm{EtOAc}(\mathbf{1})$

\begin{tabular}{|c|c|c|c|c|}
\hline$\underline{\text { Atom }} \underline{1}$ & $\underline{\text { Atom }} \underline{2}$ & $\underline{\text { Atom }} \underline{3}$ & $\underline{\text { Atom }} 4$ & Angle \\
\hline$C(51)$ & $C(5)$ & $C(6)$ & $\mathrm{N}(6)$ & $-179.16(0.46)$ \\
\hline C(51) & $C(5)$ & $\mathrm{C}(6)$ & $C(7)$ & $4.37(0.79)$ \\
\hline$C(4)$ & $C(5)$ & $C(51)$ & $C(52)$ & $-106.15(0.59)$ \\
\hline C(4) & $C(5)$ & $\mathrm{C}(51)$ & $C(56)$ & $75.98(0.68)$ \\
\hline $\mathrm{C}(6)$ & $C(5)$ & $\mathrm{C}(51)$ & $\mathrm{C}(52)$ & 76.07 ( 0.66) \\
\hline $\mathrm{C}(6)$ & $\mathrm{C}(5)$ & C(51) & $C(56)$ & $-101.80(0.62)$ \\
\hline $\mathrm{N}(6)$ & $C(6)$ & $\mathrm{C}(7)$ & $C(8)$ & $0.27(0.58)$ \\
\hline C(5) & $C(6)$ & $\mathrm{C}(7)$ & $\mathrm{C}(8)$ & $177.22(0.51)$ \\
\hline $\mathrm{C}(6)$ & $\mathrm{C}(7)$ & $\mathrm{C}(8)$ & $\mathrm{C}(9)$ & $-0.21(0.57)$ \\
\hline$C(7)$ & $C(8)$ & $C(9)$ & $\mathrm{N}(6)$ & $0.08(0.59)$ \\
\hline$C(7)$ & $C(8)$ & $\mathrm{C}(9)$ & $\mathrm{C}(10)$ & $-179.44(0.50)$ \\
\hline $\mathrm{N}(6)$ & $\mathrm{C}(9)$ & $\mathrm{C}(10)$ & $\mathrm{C}(11)$ & $-2.86(0.83)$ \\
\hline $\mathrm{N}(6)$ & $\mathrm{C}(9)$ & $\mathrm{C}(10)$ & $\mathrm{C}(101)$ & $-179.70(0.46)$ \\
\hline$C(8)$ & $C(9)$ & $C(10)$ & $\mathrm{C}(11)$ & $176.58(0.50)$ \\
\hline $\mathrm{C}(8)$ & $C(9)$ & $\mathrm{C}(10)$ & $\mathrm{C}(101)$ & $-0.26(0.79)$ \\
\hline C(9) & $\mathrm{C}(10)$ & $\mathrm{C}(11)$ & $\mathrm{N}(11)$ & $2.54(0.84)$ \\
\hline $\mathrm{C}(9)$ & $\mathrm{C}(10)$ & $\mathrm{C}(11)$ & $\mathrm{C}(12)$ & $-178.48(0.52)$ \\
\hline C(101) & $\mathrm{C}(10)$ & $\mathrm{C}(11)$ & $\mathrm{N}(11)$ & $179.43(0.46)$ \\
\hline C(101) & $\mathrm{C}(10)$ & $\mathrm{C}(11)$ & $\mathrm{C}(12)$ & $-1.59(0.78)$ \\
\hline C(9) & $\mathrm{C}(10)$ & $\mathrm{C}(101)$ & $\mathrm{C}(102)$ & $-83.25(0.66)$ \\
\hline $\mathrm{C}(9)$ & $\mathrm{C}(10)$ & $\mathrm{C}(101)$ & $\mathrm{C}(106)$ & $98.55(0.62)$ \\
\hline $\mathrm{C}(11)$ & $\mathrm{C}(10)$ & $C(101)$ & $\mathrm{C}(102)$ & $99.65(0.63)$ \\
\hline $\mathrm{C}(11)$ & $\mathrm{C}(10)$ & $\mathrm{C}(101)$ & $\mathrm{C}(106)$ & $-78.56(0.64)$ \\
\hline $\mathrm{N}(11)$ & $\mathrm{C}(11)$ & $\mathrm{C}(12)$ & $\mathrm{C}(13)$ & $-1.09(0.62)$ \\
\hline $\mathrm{C}(10)$ & $\mathrm{C}(11)$ & $\mathrm{C}(12)$ & $C(13)$ & 179.79 ( 0.53) \\
\hline $\mathrm{C}(11)$ & $\mathrm{C}(12)$ & $\mathrm{C}(13)$ & $\mathrm{C}(14)$ & $0.99(0.65)$ \\
\hline $\mathrm{C}(12)$ & $\mathrm{C}(13)$ & $\mathrm{C}(14)$ & $\mathrm{N}(11)$ & $-0.55(0.63)$ \\
\hline $\mathrm{C}(12)$ & $C(13)$ & $C(14)$ & C(15) & $-177.14(0.54)$ \\
\hline $\mathrm{N}(11)$ & $\mathrm{C}(14)$ & $\mathrm{C}(15)$ & C(16) & $0.12(0.83)$ \\
\hline $\mathrm{N}(11)$ & $\mathrm{C}(14)$ & $\mathrm{C}(15)$ & C(151) & $-177.96(0.47)$ \\
\hline $\mathrm{C}(13)$ & $\mathrm{C}(14)$ & $\mathrm{C}(15)$ & $C(16)$ & $176.20(0.55)$ \\
\hline $\mathrm{C}(13)$ & $\mathrm{C}(14)$ & $\mathrm{C}(15)$ & C(151) & $-1.89(0.81)$ \\
\hline $\mathrm{C}(14)$ & C(15) & $C(16)$ & $\mathrm{N}(16)$ & $1.88(0.84)$ \\
\hline $\mathrm{C}(14)$ & $\mathrm{C}(15)$ & $\mathrm{C}(16)$ & $\mathrm{C}(17)$ & $-170.87(0.60)$ \\
\hline C(151) & $\mathrm{C}(15)$ & $C(16)$ & $\mathrm{N}(16)$ & $179.90(0.50)$ \\
\hline C(151) & $\mathrm{C}(15)$ & $C(16)$ & C(17) & $7.15(0.95)$ \\
\hline $\mathrm{C}(14)$ & C(15) & C(151) & C(152) & $-94.15(0.66)$ \\
\hline $\mathrm{C}(14)$ & $\mathrm{C}(15)$ & $C(151)$ & C(156) & $83.24(0.69)$ \\
\hline$C(16)$ & $\mathrm{C}(15)$ & C(151) & C(152) & $87.66(0.70)$ \\
\hline $\mathrm{C}(16)$ & $\mathrm{C}(15)$ & $\mathrm{C}(151)$ & $\mathrm{C}(156)$ & $-94.94(0.70)$ \\
\hline $\mathrm{N}(16)$ & C(16) & $\mathrm{C}(17)$ & C(18) & $-1.40(0.67)$ \\
\hline $\mathrm{C}(15)$ & $\mathrm{C}(16)$ & $\mathrm{C}(17)$ & C(18) & $172.07(0.60)$ \\
\hline$C(16)$ & $\mathrm{C}(17)$ & $\mathrm{C}(18)$ & C(19) & $1.11(0.67)$ \\
\hline $\mathrm{C}(17)$ & $\mathrm{C}(18)$ & $\mathrm{C}(19)$ & $\mathrm{N}(16)$ & $-0.43(0.63)$ \\
\hline $\mathrm{C}(17)$ & $\mathrm{C}(18)$ & $\mathrm{C}(19)$ & $\mathrm{C}(1)$ & $-176.88(0.65)$ \\
\hline$C(5)$ & $\mathrm{C}(51)$ & $C(52)$ & $F(52)$ & $2.64(0.78)$ \\
\hline
\end{tabular}


Table of Torsional Angles in Degrees (cont)

for $\mathrm{Mn}(\mathrm{tpfc}) \mathrm{EtOAc} \cdot \mathrm{EtOAc}(\mathbf{1})$

\begin{tabular}{|c|c|c|c|c|}
\hline$\underline{\text { Atom }} \underline{1}$ & $\underline{\text { Atom }} \underline{2}$ & $\underline{\text { Atom }} \underline{3}$ & $\underline{\text { Atom }} 4$ & Angle \\
\hline$C(5)$ & $\mathrm{C}(51)$ & $C(52)$ & C(53) & $-177.46(0.51)$ \\
\hline C(56) & $\mathrm{C}(51)$ & $C(52)$ & $F(52)$ & $-179.36(0.49)$ \\
\hline C(56) & $\mathrm{C}(51)$ & $\mathrm{C}(52)$ & $C(53)$ & $0.53(0.81)$ \\
\hline$C(5)$ & $\mathrm{C}(51)$ & $C(56)$ & $F(56)$ & $-2.08(0.82)$ \\
\hline$C(5)$ & $\mathrm{C}(51)$ & $C(56)$ & C(55) & $177.93(0.53)$ \\
\hline C(52) & $C(51)$ & $C(56)$ & $F(56)$ & 179.95 ( 0.63) \\
\hline C(52) & $\mathrm{C}(51)$ & $C(56)$ & $C(55)$ & $-0.03(0.72)$ \\
\hline $\mathrm{F}(52)$ & $\mathrm{C}(52)$ & C(53) & $F(53)$ & $1.52(0.80)$ \\
\hline$F(52)$ & $\mathrm{C}(52)$ & C(53) & $C(54)$ & $179.47(0.51)$ \\
\hline C(51) & $\mathrm{C}(52)$ & C(53) & $F(53)$ & $-178.37(0.50)$ \\
\hline C(51) & $\mathrm{C}(52)$ & C(53) & $C(54)$ & $-0.43(0.88)$ \\
\hline $\mathrm{F}(53)$ & C(53) & $\mathrm{C}(54)$ & $F(54)$ & $-2.53(0.84)$ \\
\hline $\mathrm{F}(53)$ & C(53) & $C(54)$ & $C(55)$ & $177.74(0.54)$ \\
\hline C(52) & $C(53)$ & $C(54)$ & $F(54)$ & $179.52(0.51)$ \\
\hline C(52) & C(53) & $C(54)$ & $C(55)$ & $-0.21(0.90)$ \\
\hline $\mathrm{F}(54)$ & $\mathrm{C}(54)$ & $C(55)$ & $F(55)$ & $-1.59(0.87)$ \\
\hline $\mathrm{F}(54)$ & $\mathrm{C}(54)$ & C(55) & $C(56)$ & $-179.02(0.53)$ \\
\hline C(53) & C(54) & $\mathrm{C}(55)$ & $F(55)$ & $178.13(0.53)$ \\
\hline C(53) & $\mathrm{C}(54)$ & C(55) & $C(56)$ & $0.71(0.90)$ \\
\hline$F(55)$ & C(55) & $C(56)$ & $F(56)$ & $1.99(0.82)$ \\
\hline$F(55)$ & C(55) & $C(56)$ & $C(51)$ & $-178.02(0.52)$ \\
\hline C(54) & $C(55)$ & $C(56)$ & $F(56)$ & $179.42(0.53)$ \\
\hline C(54) & $\mathrm{C}(55)$ & $C(56)$ & $C(51)$ & $-0.60(0.92)$ \\
\hline $\mathrm{C}(10)$ & $\mathrm{C}(101)$ & $\mathrm{C}(102)$ & $\mathrm{F}(102)$ & $1.43(0.83)$ \\
\hline$C(10)$ & $C(101)$ & $C(102)$ & C(103) & $-178.47(0.61)$ \\
\hline C(106) & $C(101)$ & $C(102)$ & $\mathrm{F}(102)$ & $179.73(0.51)$ \\
\hline C(106) & $\mathrm{C}(101)$ & $\mathrm{C}(102)$ & C(103) & $-0.16(0.88)$ \\
\hline $\mathrm{C}(10)$ & $\mathrm{C}(101)$ & $C(106)$ & $F(106)$ & $-2.58(0.81)$ \\
\hline$C(10)$ & $C(101)$ & $C(106)$ & $C(105)$ & $178.09(0.54)$ \\
\hline C(102) & $C(101)$ & $C(106)$ & $\mathrm{F}(106)$ & 179.08 ( 0.52$)$ \\
\hline C(102) & $\mathrm{C}(101)$ & $C(106)$ & C(105) & $-0.25(0.84)$ \\
\hline$F(102)$ & $\mathrm{C}(102)$ & C(103) & $\mathrm{F}(103)$ & $-0.12(0.97)$ \\
\hline$F(102)$ & $C(102)$ & $C(103)$ & C(104) & $-179.76(0.58)$ \\
\hline C(101) & $C(102)$ & C(103) & $F(103)$ & 179.78 ( 0.60$)$ \\
\hline C(101) & $\mathrm{C}(102)$ & C(103) & C(104) & $0.14(1.02)$ \\
\hline$F(103)$ & $C(103)$ & $C(104)$ & $\mathrm{F}(10)$ & $-0.03(1.11)$ \\
\hline$F(103)$ & $C(103)$ & $C(104)$ & C(105) & $-179.33(0.63)$ \\
\hline C(102) & $C(103)$ & $C(104)$ & $F(10)$ & $179.63(0.60)$ \\
\hline C(102) & $C(103)$ & C(104) & C(105) & 0.31 ( 1.01) \\
\hline $\mathrm{F}(10)$ & $\mathrm{C}(104)$ & $\mathrm{C}(105)$ & $\mathrm{F}(105)$ & $-1.71(0.89)$ \\
\hline $\mathrm{F}(10)$ & $C(104)$ & C(105) & $\mathrm{C}(106)$ & 179.96 ( 0.52) \\
\hline$C(103)$ & $C(104)$ & $\mathrm{C}(105)$ & $F(105)$ & 177.61 ( 0.57) \\
\hline$C(103)$ & $C(104)$ & C(105) & $\mathrm{C}(106)$ & $-0.71(0.96)$ \\
\hline$F(105)$ & $C(105)$ & $C(106)$ & $F(106)$ & $3.04(0.86)$ \\
\hline$F(105)$ & $\mathrm{C}(105)$ & $C(106)$ & C(101) & $-177.62(0.50)$ \\
\hline$C(104)$ & $C(105)$ & $C(106)$ & $\mathrm{F}(106)$ & -178.6 \\
\hline
\end{tabular}


Table of Torsional Angles in Degrees (cont)

for $\mathrm{Mn}(\mathrm{tpfc}) \mathrm{EtOAc} \cdot \mathrm{EtOAc}(\mathbf{1})$

\begin{tabular}{|c|c|c|c|c|}
\hline Atom 1 & $\underline{\text { Atom }} \underline{2}$ & $\underline{\text { Atom }} \underline{3}$ & $\underline{\text { Atom }} 4$ & Angle \\
\hline$\overline{\mathrm{C}(104)}$ & $\overline{\mathrm{C}(105)}$ & $\overline{C(106)}$ & $\overline{C(101)}$ & $\overline{0.70(} 0.93)$ \\
\hline $\mathrm{C}(15)$ & $C(151)$ & C(152) & $F(152)$ & $-1.17(0.86)$ \\
\hline C(15) & $\mathrm{C}(151)$ & C(152) & C(153) & $175.40(0.55)$ \\
\hline$C(156)$ & $C(151)$ & C(152) & $\mathrm{F}(152)$ & $-178.63(0.54)$ \\
\hline$C(156)$ & $C(151)$ & C(152) & C(153) & $-2.06(0.89)$ \\
\hline$C(15)$ & $\mathrm{C}(151)$ & $\mathrm{C}(156)$ & $F(156)$ & $3.54(0.93)$ \\
\hline C(15) & $\mathrm{C}(151)$ & C(156) & $C(155)$ & $-177.05(0.60)$ \\
\hline$C(152)$ & $C(151)$ & C(156) & $F(156)$ & $-178.97(0.57)$ \\
\hline$C(152)$ & $C(151)$ & C(156) & C(155) & $0.44(0.95)$ \\
\hline$F(152)$ & $C(152)$ & C(153) & $\mathrm{F}(153)$ & $-0.93(0.84)$ \\
\hline $\mathrm{F}(152)$ & $C(152)$ & C(153) & C(154) & $179.52(0.54)$ \\
\hline$C(151)$ & $C(152)$ & C(153) & $\mathrm{F}(153)$ & $-177.62(0.54)$ \\
\hline$C(151)$ & $C(152)$ & C(153) & C(154) & $2.84(0.90)$ \\
\hline$F(153)$ & $\mathrm{C}(153)$ & C(154) & $\mathrm{F}(154)$ & $-0.29(0.89)$ \\
\hline$F(153)$ & $C(153)$ & C(154) & C(155) & $178.49(0.58)$ \\
\hline$C(152)$ & $C(153)$ & C(154) & $\mathrm{F}(154)$ & $179.26(0.53)$ \\
\hline$C(152)$ & $C(153)$ & C(154) & C(155) & $-1.95(0.94)$ \\
\hline $\mathrm{F}(154)$ & $C(154)$ & C(155) & F(155) & $-0.58(0.94)$ \\
\hline$F(154)$ & $C(154)$ & C(155) & $C(156)$ & 179.17 ( 0.59) \\
\hline C(153) & $C(154)$ & C(155) & $\mathrm{F}(155)$ & $-179.34(0.58)$ \\
\hline C(153) & $C(154)$ & C(155) & C(156) & 0.40 ( 0.99) \\
\hline $\mathrm{F}(155)$ & $C(155)$ & C(156) & $\mathrm{F}(156)$ & $-0.44(0.97)$ \\
\hline $\mathrm{F}(155)$ & $C(155)$ & C(156) & C(151) & $-179.87(0.63)$ \\
\hline$C(154)$ & $C(155)$ & $C(156)$ & $F(156)$ & $179.82(0.59)$ \\
\hline$C(154)$ & $C(155)$ & $C(156)$ & $C(151)$ & $0.38(1.01)$ \\
\hline
\end{tabular}


Table S2.1 CRYSTAL DATA AND DATA COLLECTION PARAMETERS FOR

(tpfc)CrNTs

\begin{tabular}{|c|c|}
\hline formula & $\mathrm{C}_{44} \mathrm{H}_{15} \mathrm{CrF}_{15} \mathrm{~N}_{5} \mathrm{O}_{2} \mathrm{~S}$ \\
\hline formula weight & 1014.68 \\
\hline space group & $\mathrm{P} 2_{1} / \mathrm{n}($ No. 14$)$ \\
\hline a, $\AA$ & $11.2455(3)$ \\
\hline $\mathrm{b}, \AA$ & $27.8795(7)$ \\
\hline c, $\AA$ & $13.7974(4)$ \\
\hline$\beta$, deg & $94.1214(9)$ \\
\hline $\mathrm{V}, \AA^{3}$ & $4314.6(2)$ \\
\hline $\mathrm{Z}$ & 4 \\
\hline $\mathrm{d}_{\text {calc }}, \mathrm{g} \mathrm{cm}^{-3}$ & 1.562 \\
\hline crystal dimensions, mm & $0.44 \times 0.25 \times 0.10$ \\
\hline temperature, $\mathrm{K}$ & 150. \\
\hline radiation (wavelength, $\AA$ ) & Mo $K_{\alpha}(0.71073)$ \\
\hline monochromator & graphite \\
\hline linear abs coef, mm-1 & 0.410 \\
\hline absorption correction applied & empirical $^{\mathrm{a}}$ \\
\hline transmission factors: min, max & $0.93,0.96$ \\
\hline diffractometer & Nonius KappaCCD \\
\hline h, k, l range & 0 to $14 \quad 0$ to $34-17$ to 17 \\
\hline $2 \theta$ range, deg & $4.16-52.68$ \\
\hline mosaicity, deg & 0.97 \\
\hline programs used & SHELXTL \\
\hline $\begin{array}{l}\mathrm{F}_{000} \\
\text { weighting }\end{array}$ & 2020.0 \\
\hline $1 /\left[\sigma^{2}\left(\mathrm{Fo}^{2}\right)+(0.0753 \mathrm{P})^{2}+16.9965 \mathrm{P}\right]$ where $\mathrm{P}=\left(\mathrm{Fo}^{2}+2 \mathrm{Fc}^{2}\right) / 3$ & \\
\hline data collected & 27140 \\
\hline unique data & 6737 \\
\hline $\mathrm{R}_{\text {int }}$ & 0.050 \\
\hline data used in refinement & 6737 \\
\hline cutoff used in R-factor calculations & $\mathrm{F}_{\mathrm{o}}^{2}>2.0 \sigma\left(\mathrm{F}_{\mathrm{o}}^{2}\right)$ \\
\hline data with $\mathrm{I}>2.0 \sigma(\mathrm{I})$ & 5590 \\
\hline number of variables & 614 \\
\hline largest shift/esd in final cycle & 0.00 \\
\hline $\mathrm{R}\left(\mathrm{F}_{0}\right)$ & 0.079 \\
\hline $\mathrm{R}_{\mathrm{w}}\left(\mathrm{F}_{0}^{2}\right)$ & 0.189 \\
\hline goodness of fit & 1.114 \\
\hline
\end{tabular}

${ }^{\mathrm{a}}$ Otwinowski Z. \& Minor, W. Methods Enzymol. 1996,276307. 
Table S2.2 Table of Bond Distances in Angstroms

for (tpfc)CrNTs

\begin{tabular}{|c|c|c|c|c|c|}
\hline Atom 1 & Atom 2 & Distance & Atom 1 & Atom 2 & Distance \\
\hline$\overline{\mathrm{Cr}}$ & $\mathrm{N} 20$ & $\overline{1.653(5)}$ & $\overline{\mathrm{C} 10}$ & $\overline{\mathrm{C} 11}$ & $\overline{1.387(8)}$ \\
\hline $\mathrm{Cr}$ & N1 & $1.919(4)$ & C10 & C101 & $1.507(7)$ \\
\hline $\mathrm{Cr}$ & N6 & $1.940(4)$ & C11 & C12 & $1.443(7)$ \\
\hline $\mathrm{Cr}$ & N16 & $1.942(4)$ & C12 & C13 & $1.358(8)$ \\
\hline $\mathrm{Cr}$ & N11 & $1.968(4)$ & C12 & H12 & $0.950(8)$ \\
\hline S20 & $\mathrm{O} 21$ & $1.403(5)$ & C13 & C14 & 1.433(8) \\
\hline S20 & $\mathrm{O} 22$ & $1.425(5)$ & C13 & H13 & $0.950(8)$ \\
\hline S20 & N20 & $1.680(5)$ & C14 & C15 & $1.404(7)$ \\
\hline S20 & C21 & $1.781(6)$ & C15 & C16 & $1.384(7)$ \\
\hline F52 & C52 & $1.338(6)$ & C15 & C151 & $1.496(7)$ \\
\hline F53 & C53 & $1.341(7)$ & C16 & C17 & $1.417(7)$ \\
\hline F54 & C54 & $1.355(7)$ & C17 & C18 & $1.364(8)$ \\
\hline F55 & C55 & $1.347(7)$ & C17 & H17 & $0.950(8)$ \\
\hline F56 & C56 & $1.336(7)$ & C18 & C19 & $1.406(8)$ \\
\hline F102 & C102 & $1.331(7)$ & C18 & H18 & $0.950(8)$ \\
\hline F103 & C103 & $1.342(7)$ & C21 & C22 & $1.331(9)$ \\
\hline F104 & C104 & $1.334(7)$ & C21 & C26 & $1.385(9)$ \\
\hline F105 & C105 & $1.363(7)$ & C22 & C23 & $1.369(9)$ \\
\hline F106 & C106 & $1.354(6)$ & C22 & H22 & $0.950(9)$ \\
\hline F152 & C152 & $1.345(7)$ & C23 & C24 & $1.372(9)$ \\
\hline F153 & C153 & $1.354(7)$ & C23 & $\mathrm{H} 23$ & $0.950(9)$ \\
\hline F154 & C154 & $1.334(6)$ & C24 & C25 & $1.377(9)$ \\
\hline F155 & C155 & $1.345(6)$ & C24 & C27 & $1.521(8)$ \\
\hline F156 & C156 & $1.335(6)$ & C25 & C26 & $1.434(9)$ \\
\hline N1 & C1 & $1.364(7)$ & C25 & $\mathrm{H} 25$ & $0.950(9)$ \\
\hline N1 & C4 & $1.390(7)$ & C26 & H26 & $0.950(9)$ \\
\hline N6 & C9 & $1.389(7)$ & C27 & H27A & $0.980(9)$ \\
\hline N6 & C6 & $1.408(7)$ & C27 & H27B & $0.980(9)$ \\
\hline N11 & C11 & $1.376(7)$ & C27 & H27C & $0.980(9)$ \\
\hline N11 & C14 & $1.387(7)$ & C51 & C52 & $1.372(8)$ \\
\hline N16 & C19 & $1.367(7)$ & C51 & C56 & $1.385(8)$ \\
\hline N16 & C16 & $1.378(6)$ & C52 & C53 & $1.379(8)$ \\
\hline C1 & C19 & $1.400(7)$ & C53 & C54 & $1.359(9)$ \\
\hline C1 & C2 & $1.420(7)$ & C54 & C55 & $1.367(10)$ \\
\hline C2 & C3 & $1.364(8)$ & C55 & C56 & $1.379(9)$ \\
\hline C2 & H2 & $0.950(8)$ & C101 & C106 & $1.377(8)$ \\
\hline C3 & C4 & $1.419(8)$ & C101 & C102 & 1.382(8) \\
\hline C3 & H3 & $0.950(8)$ & C102 & C103 & $1.385(8)$ \\
\hline C4 & C5 & $1.377(8)$ & C103 & C104 & $1.373(9)$ \\
\hline C5 & C6 & $1.410(8)$ & C104 & C105 & $1.357(9)$ \\
\hline C5 & C51 & $1.499(7)$ & C105 & C106 & $1.373(8)$ \\
\hline C6 & C7 & $1.417(8)$ & C151 & C156 & $1.386(8)$ \\
\hline C7 & C8 & $1.371(8)$ & C151 & C152 & $1.403(8)$ \\
\hline C7 & H7 & $0.950(8)$ & C152 & C153 & $1.370(8)$ \\
\hline C8 & C9 & $1.442(7)$ & C153 & C154 & 1.356(9) \\
\hline C8 & H8 & $0.950(7)$ & C154 & C155 & $1.369(9)$ \\
\hline C9 & C10 & $1.376(8)$ & C155 & C156 & $1.363(8)$ \\
\hline
\end{tabular}

Numbers in parentheses are estimated standard deviations in the least significant digits. 
Table S2.3. Table of Bond Angles in Degrees

for (tpfc)CrNTs

\begin{tabular}{|c|c|c|c|c|c|c|c|}
\hline Atom 1 & Atom $\underline{2}$ & $\underline{\text { Atom }} \underline{3}$ & Angle & $\underline{\text { Atom }} \underline{1}$ & $\underline{\text { Atom }} \underline{2}$ & $\underline{\text { Atom }} \underline{3}$ & Angle \\
\hline$\overline{\mathrm{N} 20}$ & $\overline{\mathrm{Cr}}$ & $\mathrm{N} 1$ & $1 \overline{02.9(2)}$ & $\overline{\mathrm{C} 8}$ & $\overline{\mathrm{C} 7}$ & $\overline{\mathrm{H} 7}$ & $1 \overline{25.6(5)}$ \\
\hline N20 & $\mathrm{Cr}$ & N6 & $104.2(2)$ & C6 & C7 & H7 & $125.6(5)$ \\
\hline N1 & $\mathrm{Cr}$ & N6 & 86.58(18) & C7 & C8 & C9 & $106.9(5)$ \\
\hline N20 & $\mathrm{Cr}$ & N16 & $108.6(2)$ & C7 & C8 & H8 & $126.6(5)$ \\
\hline N1 & $\mathrm{Cr}$ & N16 & 77.39(18) & C9 & C8 & H8 & $126.6(5)$ \\
\hline N6 & $\mathrm{Cr}$ & N16 & $145.92(18)$ & C10 & C9 & N6 & 123.5(5) \\
\hline N20 & $\mathrm{Cr}$ & N11 & $112.2(2)$ & C10 & C9 & C8 & $127.2(5)$ \\
\hline N1 & $\mathrm{Cr}$ & N11 & 144.32(18) & N6 & C9 & C8 & 109.1(4) \\
\hline N6 & $\mathrm{Cr}$ & N11 & $91.26(18)$ & C9 & C10 & C11 & $126.2(5)$ \\
\hline N16 & $\mathrm{Cr}$ & N11 & $85.00(17)$ & C9 & C10 & C101 & $117.8(5)$ \\
\hline $\mathrm{O} 21$ & S20 & $\mathrm{O} 22$ & 118.2(3) & C11 & C10 & C101 & $115.7(5)$ \\
\hline $\mathrm{O} 21$ & S20 & N20 & 105.8(3) & N11 & C11 & C10 & 124.1(5) \\
\hline $\mathrm{O} 22$ & S20 & N20 & 109.7(3) & N11 & C11 & C12 & 108.7(4) \\
\hline $\mathrm{O} 21$ & S20 & C21 & $110.6(3)$ & C10 & C11 & C12 & 127.1(5) \\
\hline $\mathrm{O} 22$ & S20 & C21 & 109.3(3) & C13 & C12 & C11 & 107.1(5) \\
\hline N20 & S20 & C21 & 101.9(3) & C13 & C12 & H12 & $126.5(5)$ \\
\hline C1 & N1 & C4 & 108.8(4) & C11 & C12 & H12 & $126.5(5)$ \\
\hline C1 & N1 & $\mathrm{Cr}$ & 118.1(3) & C12 & C13 & C14 & 108.3(5) \\
\hline C4 & N1 & $\mathrm{Cr}$ & $132.6(4)$ & C12 & C13 & H13 & $125.8(5)$ \\
\hline C9 & N6 & C6 & $106.7(4)$ & C14 & C13 & H13 & 125.8(5) \\
\hline C9 & N6 & $\mathrm{Cr}$ & 124.4(3) & N11 & C14 & C15 & 125.7(5) \\
\hline C6 & N6 & $\mathrm{Cr}$ & $127.5(3)$ & N11 & C14 & C13 & 108.1(5) \\
\hline C11 & N11 & C14 & $107.8(4)$ & C15 & C14 & C13 & $126.0(5)$ \\
\hline C11 & N11 & $\mathrm{Cr}$ & 123.3(3) & C16 & C15 & C14 & $122.0(5)$ \\
\hline C14 & N11 & $\mathrm{Cr}$ & $126.8(4)$ & C16 & C15 & C151 & $118.9(4)$ \\
\hline C19 & N16 & C16 & $108.3(4)$ & C14 & C15 & C151 & 118.9(5) \\
\hline C19 & N16 & $\mathrm{Cr}$ & $117.4(3)$ & N16 & C16 & C15 & $120.6(5)$ \\
\hline C16 & N16 & $\mathrm{Cr}$ & 133.7(4) & N16 & C16 & C17 & $107.5(4)$ \\
\hline $\mathrm{Cr}$ & N20 & S20 & 150.7(3) & C15 & C16 & C17 & 131.8(5) \\
\hline N1 & C1 & C19 & 112.1(5) & C18 & C17 & C16 & $108.0(5)$ \\
\hline N1 & C1 & C2 & $108.5(4)$ & C18 & C17 & H17 & $126.0(5)$ \\
\hline C19 & $\mathrm{C} 1$ & $\mathrm{C} 2$ & 139.1(5) & C16 & C17 & H17 & $126.0(5)$ \\
\hline C3 & $\mathrm{C} 2$ & C1 & 107.1(5) & C17 & C18 & C19 & 107.5(5) \\
\hline C3 & C2 & $\mathrm{H} 2$ & $126.5(5)$ & C17 & C18 & H18 & $126.3(5)$ \\
\hline C1 & C2 & $\mathrm{H} 2$ & $126.5(5)$ & C19 & C18 & H18 & 126.3(5) \\
\hline C2 & C3 & C4 & 108.9(5) & N16 & C19 & C1 & $111.5(4)$ \\
\hline C2 & C3 & H3 & $125.6(5)$ & N16 & C19 & C18 & 108.7(5) \\
\hline C4 & C3 & H3 & $125.6(5)$ & C1 & C19 & C18 & 139.5(5) \\
\hline C5 & C4 & N1 & $120.8(5)$ & C22 & C21 & C26 & $123.9(6)$ \\
\hline C5 & C4 & C3 & 132.3(5) & C22 & C21 & S20 & 119.1(5) \\
\hline N1 & C4 & C3 & 106.7(5) & C26 & C21 & S20 & $116.9(5)$ \\
\hline C4 & C5 & C6 & $123.4(5)$ & C21 & C22 & C23 & $118.3(7)$ \\
\hline C4 & C5 & C51 & $118.0(5)$ & C21 & C22 & H22 & $120.8(7)$ \\
\hline C6 & C5 & C51 & 118.5(5) & C23 & C22 & H22 & $120.8(7)$ \\
\hline N6 & C6 & $\mathrm{C} 5$ & 123.6(5) & C22 & C23 & $\mathrm{C} 24$ & $122.0(7)$ \\
\hline N6 & C6 & C7 & $108.6(4)$ & C22 & C23 & H23 & $119.0(7)$ \\
\hline C5 & C6 & C7 & $127.6(5)$ & C24 & C23 & H23 & $119.0(7)$ \\
\hline C8 & C7 & C6 & 108.7(5) & C23 & C24 & C25 & $119.9(6)$ \\
\hline
\end{tabular}


Bond Angles (cont.)

\begin{tabular}{|c|c|c|c|c|c|c|c|}
\hline Atom 1 & $\underline{\text { Atom }} \underline{2}$ & $\underline{\text { Atom }} \underline{3}$ & $\underline{\text { Angle }}$ & Atom 1 & $\underline{\text { Atom }} \underline{2}$ & $\underline{\text { Atom }} \underline{3}$ & $\underline{\text { Angle }}$ \\
\hline$\overline{\mathrm{C} 23}$ & $\overline{\mathrm{C} 24}$ & $\overline{\mathrm{C} 27}$ & $1 \overline{18.6(6)}$ & $\overline{\mathrm{C} 102}$ & $\overline{\mathrm{C} 101}$ & $\overline{\mathrm{C} 10}$ & $1 \overline{23.2(5)}$ \\
\hline C25 & C24 & C27 & 121.5(6) & F102 & C102 & C101 & 119.9(5) \\
\hline C24 & C25 & C26 & 118.9(6) & F102 & C102 & C103 & 118.5(5) \\
\hline C24 & C25 & H25 & $120.6(6)$ & C101 & C102 & C103 & $121.5(6)$ \\
\hline C26 & C25 & $\mathrm{H} 25$ & $120.6(6)$ & F103 & C103 & C104 & $120.4(5)$ \\
\hline C21 & C26 & C25 & $117.0(6)$ & F103 & C103 & C102 & $119.8(6)$ \\
\hline C21 & C26 & H26 & $121.5(6)$ & C104 & C103 & C102 & $119.7(5)$ \\
\hline C25 & C26 & H26 & $121.5(6)$ & F104 & C104 & C105 & $120.3(6)$ \\
\hline C24 & C27 & H27A & 109.5(6) & F104 & C104 & C103 & $120.0(5)$ \\
\hline C24 & C27 & H27B & 109.5(6) & C105 & C104 & C103 & 119.7(5) \\
\hline H27A & C27 & H27B & 109.5(6) & C104 & C105 & F105 & $120.4(5)$ \\
\hline $\mathrm{C} 24$ & $\mathrm{C} 27$ & H27C & 109.5(6) & C104 & C105 & C106 & $120.1(6)$ \\
\hline H27A & C27 & H27C & $109.5(6)$ & F105 & C105 & C106 & 119.5(5) \\
\hline H27B & C27 & H27C & 109.5(6) & F106 & C106 & C105 & $117.9(5)$ \\
\hline C52 & C51 & C56 & $116.7(5)$ & F106 & C106 & C101 & $119.9(5)$ \\
\hline C52 & C51 & C5 & $121.5(5)$ & C105 & C106 & C101 & $122.2(5)$ \\
\hline C56 & C51 & C5 & 121.7(5) & C156 & C151 & C152 & $116.2(5)$ \\
\hline F52 & C52 & C51 & 119.8(5) & C156 & C151 & C15 & 121.5(5) \\
\hline F52 & C52 & C53 & $118.2(5)$ & C152 & C151 & C15 & $122.2(5)$ \\
\hline C51 & C52 & C53 & $122.0(5)$ & F152 & C152 & C153 & 119.5(5) \\
\hline F53 & C53 & C54 & $120.0(5)$ & F152 & C152 & C151 & 119.3(5) \\
\hline F53 & C53 & C52 & $120.5(6)$ & C153 & C152 & C151 & 121.1(5) \\
\hline C54 & C53 & C52 & $119.5(6)$ & F153 & C153 & C154 & 119.8(5) \\
\hline F54 & C54 & C53 & $120.2(6)$ & F153 & C153 & C152 & 119.5(6) \\
\hline F54 & C54 & C55 & $119.0(6)$ & C154 & C153 & C152 & $120.7(6)$ \\
\hline C53 & C54 & C55 & $120.9(5)$ & F154 & C154 & C153 & 119.3(6) \\
\hline F55 & C55 & C54 & 121.1(6) & F154 & C154 & C155 & 121.1(5) \\
\hline F55 & C55 & C56 & $120.2(7)$ & C153 & C154 & C155 & 119.6(5) \\
\hline C54 & C55 & C56 & $118.6(6)$ & F155 & C155 & C156 & $120.9(6)$ \\
\hline F56 & C56 & C55 & $118.5(6)$ & F155 & C155 & C154 & $118.8(5)$ \\
\hline F56 & C56 & C51 & $119.2(5)$ & C156 & C155 & C154 & $120.3(5)$ \\
\hline C55 & C56 & C51 & 122.3(6) & F156 & C156 & C155 & 118.6(5) \\
\hline C106 & C101 & C102 & $116.7(5)$ & F156 & C156 & C151 & 119.3(5) \\
\hline C106 & C101 & C10 & $120.0(5)$ & C155 & C156 & C151 & $122.0(5)$ \\
\hline
\end{tabular}

Numbers in parentheses are estimated standard deviations in the least significant digits. 
Table S2.4. Table of Torsional Angles in Degrees

for (tpfc)CrNTs

\begin{tabular}{|c|c|c|c|c|}
\hline$\underline{\text { Atom }} \underline{1}$ & $\underline{\text { Atom }} \underline{2}$ & $\underline{\text { Atom }} \underline{3}$ & $\underline{\text { Atom }} \underline{4}$ & Angle \\
\hline $\mathrm{N}(6)$ & $\overline{\mathrm{Cr}}$ & $\mathrm{N}(1)$ & $\mathrm{C}(1)$ & $-165.87(0.42)$ \\
\hline$N(6)$ & $\mathrm{Cr}$ & $\mathrm{N}(1)$ & $C(4)$ & $23.23(0.50)$ \\
\hline $\mathrm{N}(11)$ & $\mathrm{Cr}$ & $\mathrm{N}(1)$ & $\mathrm{C}(1)$ & $-78.44(0.51)$ \\
\hline $\mathrm{N}(11)$ & $\mathrm{Cr}$ & $\mathrm{N}(1)$ & $C(4)$ & $110.67(0.52)$ \\
\hline $\mathrm{N}(16)$ & $\mathrm{Cr}$ & $\mathrm{N}(1)$ & $C(1)$ & -16.16 ( 0.39) \\
\hline $\mathrm{N}(16)$ & $\mathrm{Cr}$ & $\mathrm{N}(1)$ & $C(4)$ & $172.94(0.53)$ \\
\hline $\mathrm{N}(20)$ & $\mathrm{Cr}$ & $\mathrm{N}(1)$ & $\mathrm{C}(1)$ & $90.34(0.42)$ \\
\hline $\mathrm{N}(20)$ & $\mathrm{Cr}$ & $\mathrm{N}(1)$ & $\mathrm{C}(4)$ & $-80.56(0.52)$ \\
\hline $\mathrm{N}(1)$ & $\mathrm{Cr}$ & $\mathrm{N}(6)$ & $C(6)$ & $-24.37(0.45)$ \\
\hline $\mathrm{N}(1)$ & $\mathrm{Cr}$ & $\mathrm{N}(6)$ & $\mathrm{C}(9)$ & $171.07(0.44)$ \\
\hline $\mathrm{N}(11)$ & $\mathrm{Cr}$ & $\mathrm{N}(6)$ & $C(6)$ & $-168.70(0.45)$ \\
\hline $\mathrm{N}(11)$ & $\mathrm{Cr}$ & $\mathrm{N}(6)$ & $C(9)$ & $26.74(0.44)$ \\
\hline $\mathrm{N}(16)$ & $\mathrm{Cr}$ & $\mathrm{N}(6)$ & $C(6)$ & $-85.78(0.54)$ \\
\hline $\mathrm{N}(16)$ & $\mathrm{Cr}$ & $\mathrm{N}(6)$ & $C(9)$ & $109.66(0.47)$ \\
\hline $\mathrm{N}(20)$ & $\mathrm{Cr}$ & $\mathrm{N}(6)$ & $C(6)$ & $78.04(0.47)$ \\
\hline $\mathrm{N}(20)$ & $\mathrm{Cr}$ & $\mathrm{N}(6)$ & $\mathrm{C}(9)$ & $-86.52(0.45)$ \\
\hline $\mathrm{N}(1)$ & $\mathrm{Cr}$ & $\mathrm{N}(11)$ & $\mathrm{C}(11)$ & $-112.96(0.45)$ \\
\hline $\mathrm{N}(1)$ & $\mathrm{Cr}$ & $\mathrm{N}(11)$ & $\mathrm{C}(14)$ & 85.55 ( 0.53) \\
\hline $\mathrm{N}(6)$ & $\mathrm{Cr}$ & $\mathrm{N}(11)$ & $\mathrm{C}(11)$ & $-27.03(0.44)$ \\
\hline $\mathrm{N}(6)$ & $\mathrm{Cr}$ & $\mathrm{N}(11)$ & $C(14)$ & $171.48(0.45)$ \\
\hline $\mathrm{N}(16)$ & $\mathrm{Cr}$ & $\mathrm{N}(11)$ & $\mathrm{C}(11)$ & $-173.08(0.44)$ \\
\hline $\mathrm{N}(16)$ & $\mathrm{Cr}$ & $\mathrm{N}(11)$ & $\mathrm{C}(14)$ & $25.42(0.45)$ \\
\hline $\mathrm{N}(20)$ & $\mathrm{Cr}$ & $\mathrm{N}(11)$ & $\mathrm{C}(11)$ & 78.87 ( 0.46) \\
\hline $\mathrm{N}(20)$ & $\mathrm{Cr}$ & $\mathrm{N}(11)$ & $\mathrm{C}(14)$ & $-82.62(0.47)$ \\
\hline $\mathrm{N}(1)$ & $\mathrm{Cr}$ & $\mathrm{N}(16)$ & $C(16)$ & $-172.77(0.51)$ \\
\hline $\mathrm{N}(1)$ & $\mathrm{Cr}$ & $\mathrm{N}(16)$ & $\mathrm{C}(19)$ & $16.76(0.39)$ \\
\hline $\mathrm{N}(6)$ & $\mathrm{Cr}$ & $\mathrm{N}(16)$ & $C(16)$ & $-108.84(0.51)$ \\
\hline $\mathrm{N}(6)$ & $\mathrm{Cr}$ & $\mathrm{N}(16)$ & $\mathrm{C}(19)$ & $80.69(0.51)$ \\
\hline $\mathrm{N}(11)$ & $\mathrm{Cr}$ & $\mathrm{N}(16)$ & $C(16)$ & $-24.00(0.48)$ \\
\hline $\mathrm{N}(11)$ & $\mathrm{Cr}$ & $\mathrm{N}(16)$ & $\mathrm{C}(19)$ & $165.53(0.42)$ \\
\hline $\mathrm{N}(20)$ & $\mathrm{Cr}$ & $\mathrm{N}(16)$ & $C(16)$ & $87.73(0.51)$ \\
\hline $\mathrm{N}(20)$ & $\mathrm{Cr}$ & $\mathrm{N}(16)$ & $\mathrm{C}(19)$ & $-82.75(0.43)$ \\
\hline $\mathrm{N}(1)$ & $\mathrm{Cr}$ & $\mathrm{N}(20)$ & $S(20)$ & 24.98 ( 0.61) \\
\hline $\mathrm{N}(6)$ & $\mathrm{Cr}$ & $\mathrm{N}(20)$ & $S(20)$ & $-64.72(0.60)$ \\
\hline $\mathrm{N}(11)$ & $\mathrm{Cr}$ & $\mathrm{N}(20)$ & $S(20)$ & $-162.07(0.52)$ \\
\hline $\mathrm{N}(16)$ & $\mathrm{Cr}$ & $\mathrm{N}(20)$ & $S(20)$ & $105.79(0.57)$ \\
\hline $\mathrm{O}(21)$ & $\mathrm{S}(20)$ & $\mathrm{N}(20)$ & $\mathrm{Cr}$ & $-167.79(0.55)$ \\
\hline $\mathrm{O}(22)$ & $S(20)$ & $\mathrm{N}(20)$ & $\mathrm{Cr}$ & $-39.23(0.65)$ \\
\hline $\mathrm{C}(21)$ & $S(20)$ & $\mathrm{N}(20)$ & $\mathrm{Cr}$ & 76.47 ( 0.60) \\
\hline $\mathrm{O}(21)$ & $S(20)$ & $\mathrm{C}(21)$ & $\mathrm{C}(22)$ & $120.48(0.50)$ \\
\hline $\mathrm{O}(21)$ & $S(20)$ & $\mathrm{C}(21)$ & $C(26)$ & $-57.81(0.55)$ \\
\hline $\mathrm{O}(22)$ & $S(20)$ & $\mathrm{C}(21)$ & $\mathrm{C}(22)$ & -11.32 ( 0.59) \\
\hline $\mathrm{O}(22)$ & $S(20)$ & $\mathrm{C}(21)$ & $C(26)$ & $170.38(0.49)$ \\
\hline$N(20)$ & $S(20)$ & $\mathrm{C}(21)$ & $\mathrm{C}(22)$ & $-127.34(0.49)$ \\
\hline$N(20)$ & $S(20)$ & $\mathrm{C}(21)$ & $C(26)$ & $54.36(0.53)$ \\
\hline
\end{tabular}


Table of Torsional Angles in Degrees (cont)

for (tpfc)CrNTs

\begin{tabular}{|c|c|c|c|c|}
\hline$\underline{\text { Atom }} 1$ & Atom $\underline{2}$ & Atom $\underline{3}$ & $\underline{\text { Atom }} 4$ & Angle \\
\hline $\mathrm{Cr}$ & $\mathrm{N}(1)$ & $\mathrm{C}(1)$ & $\mathrm{C}(2)$ & $-172.35(0.36)$ \\
\hline $\mathrm{Cr}$ & $\mathrm{N}(1)$ & $\mathrm{C}(1)$ & C(19) & $13.00(0.62)$ \\
\hline $\mathrm{C}(4)$ & $\mathrm{N}(1)$ & $\mathrm{C}(1)$ & $C(2)$ & $0.58(0.61)$ \\
\hline C(4) & $\mathrm{N}(1)$ & $\mathrm{C}(1)$ & C(19) & $-174.08(0.47)$ \\
\hline $\mathrm{Cr}$ & $\mathrm{N}(1)$ & $\mathrm{C}(4)$ & $\mathrm{C}(3)$ & 170.11 ( 0.39) \\
\hline $\mathrm{Cr}$ & $\mathrm{N}(1)$ & $\mathrm{C}(4)$ & $\mathrm{C}(5)$ & $-13.89(0.82)$ \\
\hline $\mathrm{C}(1)$ & $\mathrm{N}(1)$ & $\mathrm{C}(4)$ & $\mathrm{C}(3)$ & $-1.41(0.60)$ \\
\hline$C(1)$ & $\mathrm{N}(1)$ & $\mathrm{C}(4)$ & $\mathrm{C}(5)$ & $174.59(0.52)$ \\
\hline $\mathrm{Cr}$ & $\mathrm{N}(6)$ & $\mathrm{C}(6)$ & $\mathrm{C}(5)$ & 18.62 ( 0.77) \\
\hline $\mathrm{Cr}$ & $\mathrm{N}(6)$ & $C(6)$ & $\mathrm{C}(7)$ & $-166.16(0.37)$ \\
\hline $\mathrm{C}(9)$ & $N(6)$ & C(6) & $\mathrm{C}(5)$ & $-174.64(0.53)$ \\
\hline C(9) & $\mathrm{N}(6)$ & C(6) & $C(7)$ & $0.58(0.59)$ \\
\hline $\mathrm{Cr}$ & $\mathrm{N}(6)$ & $\mathrm{C}(9)$ & $\mathrm{C}(8)$ & $166.32(0.36)$ \\
\hline $\mathrm{Cr}$ & $\mathrm{N}(6)$ & $\mathrm{C}(9)$ & $\mathrm{C}(10)$ & $-18.22(0.76)$ \\
\hline$C(6)$ & $N(6)$ & $\mathrm{C}(9)$ & $C(8)$ & $-0.93(0.59)$ \\
\hline C(6) & $\mathrm{N}(6)$ & $\mathrm{C}(9)$ & $\mathrm{C}(10)$ & $174.53(0.53)$ \\
\hline $\mathrm{Cr}$ & $\mathrm{N}(11)$ & $\mathrm{C}(11)$ & $\mathrm{C}(10)$ & 19.07 ( 0.76) \\
\hline $\mathrm{Cr}$ & $\mathrm{N}(11)$ & $\mathrm{C}(11)$ & C(12) & $-164.54(0.39)$ \\
\hline$C(14)$ & $\mathrm{N}(11)$ & $\mathrm{C}(11)$ & $\mathrm{C}(10)$ & $-176.40(0.53)$ \\
\hline$C(14)$ & $\mathrm{N}(11)$ & $\mathrm{C}(11)$ & $\mathrm{C}(12)$ & $-0.02(0.67)$ \\
\hline $\mathrm{Cr}$ & $\mathrm{N}(11)$ & $\mathrm{C}(14)$ & $\mathrm{C}(13)$ & $163.60(0.40)$ \\
\hline $\mathrm{Cr}$ & $\mathrm{N}(11)$ & $C(14)$ & $C(15)$ & $-20.93(0.80)$ \\
\hline $\mathrm{C}(11)$ & $\mathrm{N}(11)$ & $C(14)$ & C(13) & $-0.23(0.61)$ \\
\hline $\mathrm{C}(11)$ & $\mathrm{N}(11)$ & $\mathrm{C}(14)$ & C(15) & $175.25(0.54)$ \\
\hline $\mathrm{Cr}$ & $\mathrm{N}(16)$ & $C(16)$ & C(15) & $14.48(0.75)$ \\
\hline $\mathrm{Cr}$ & $\mathrm{N}(16)$ & $C(16)$ & $\mathrm{C}(17)$ & $-169.48(0.40)$ \\
\hline C(19) & $\mathrm{N}(16)$ & $C(16)$ & $\mathrm{C}(15)$ & $-174.42(0.48)$ \\
\hline C(19) & $\mathrm{N}(16)$ & $\mathrm{C}(16)$ & $\mathrm{C}(17)$ & $1.62(0.57)$ \\
\hline $\mathrm{Cr}$ & $\mathrm{N}(16)$ & $\mathrm{C}(19)$ & $\mathrm{C}(1)$ & $-14.55(0.61)$ \\
\hline $\mathrm{Cr}$ & $\mathrm{N}(16)$ & C(19) & $\mathrm{C}(18)$ & $171.22(0.37)$ \\
\hline$C(16)$ & $\mathrm{N}(16)$ & $C(19)$ & $\mathrm{C}(1)$ & $172.68(0.45)$ \\
\hline$C(16)$ & $\mathrm{N}(16)$ & $\mathrm{C}(19)$ & $\mathrm{C}(18)$ & $-1.55(0.60)$ \\
\hline $\mathrm{N}(1)$ & $\mathrm{C}(1)$ & $\mathrm{C}(2)$ & $\mathrm{C}(3)$ & $0.53(0.64)$ \\
\hline C(19) & $\mathrm{C}(1)$ & $\mathrm{C}(2)$ & C(3) & $172.96(0.70)$ \\
\hline $\mathrm{N}(1)$ & $\mathrm{C}(1)$ & $\mathrm{C}(19)$ & $\mathrm{N}(16)$ & $1.10(0.68)$ \\
\hline $\mathrm{N}(1)$ & $\mathrm{C}(1)$ & $\mathrm{C}(19)$ & $\mathrm{C}(18)$ & $172.67(0.67)$ \\
\hline $\mathrm{C}(2)$ & $\mathrm{C}(1)$ & C(19) & $\mathrm{N}(16)$ & $-171.14(0.67)$ \\
\hline$C(2)$ & $\mathrm{C}(1)$ & C(19) & $\mathrm{C}(18)$ & $0.43(1.35)$ \\
\hline $\mathrm{C}(1)$ & $\mathrm{C}(2)$ & $\mathrm{C}(3)$ & $\mathrm{C}(4)$ & $-1.41(0.65)$ \\
\hline $\mathrm{C}(2)$ & $\mathrm{C}(3)$ & $\mathrm{C}(4)$ & $\mathrm{N}(1)$ & $1.76(0.64)$ \\
\hline $\mathrm{C}(2)$ & $\mathrm{C}(3)$ & $\mathrm{C}(4)$ & $\mathrm{C}(5)$ & $-173.60(0.61)$ \\
\hline $\mathrm{N}(1)$ & $C(4)$ & C(5) & $C(6)$ & $-3.27(0.87)$ \\
\hline $\mathrm{N}(1)$ & $C(4)$ & $C(5)$ & $\mathrm{C}(51)$ & $-178.36(0.49)$ \\
\hline C(3) & $\mathrm{C}(4)$ & $\mathrm{C}(5)$ & $\mathrm{C}(6)$ & $171.54(0.59)$ \\
\hline C(3) & $\mathrm{C}(4)$ & C(5) & C(51) & $-3.55(0.95)$ \\
\hline C(4) & C(5) & $C(6)$ & $\mathrm{N}(6)$ & $0.37(0.89)$ \\
\hline
\end{tabular}


Table of Torsional Angles in Degrees (cont)

for (tpfc)CrNTs

\begin{tabular}{|c|c|c|c|c|}
\hline$\underline{\text { Atom }} \underline{1}$ & $\underline{\text { Atom }} \underline{2}$ & $\underline{\text { Atom }} \underline{3}$ & Atom 4 & Angle \\
\hline$\overline{C(4)}$ & $\overline{\mathrm{C}(5)}$ & $\overline{\mathrm{C}(6)}$ & $\overline{\mathrm{C}(7)}$ & $-\overline{73.91}(0.57)$ \\
\hline C(51) & $C(5)$ & $C(6)$ & $\mathrm{N}(6)$ & $175.45(0.50)$ \\
\hline C(51) & $C(5)$ & $C(6)$ & $C(7)$ & $1.17(0.88)$ \\
\hline$C(4)$ & $C(5)$ & $C(51)$ & $C(52)$ & $91.85(0.68)$ \\
\hline C(4) & $\mathrm{C}(5)$ & $\mathrm{C}(51)$ & $\mathrm{C}(56)$ & $-84.14(0.72)$ \\
\hline$C(6)$ & $\mathrm{C}(5)$ & $\mathrm{C}(51)$ & $\mathrm{C}(52)$ & $-83.49(0.70)$ \\
\hline$C(6)$ & $C(5)$ & $C(51)$ & $C(56)$ & $100.52(0.68)$ \\
\hline $\mathrm{N}(6)$ & $C(6)$ & $\mathrm{C}(7)$ & $C(8)$ & $0.00(0.77)$ \\
\hline$C(5)$ & $C(6)$ & $\mathrm{C}(7)$ & $C(8)$ & $174.99(0.56)$ \\
\hline $\mathrm{C}(6)$ & $\mathrm{C}(7)$ & $\mathrm{C}(8)$ & $\mathrm{C}(9)$ & $-0.58(0.64)$ \\
\hline C(7) & $C(8)$ & $C(9)$ & $\mathrm{N}(6)$ & $0.95(0.63)$ \\
\hline$C(7)$ & $C(8)$ & $\mathrm{C}(9)$ & $\mathrm{C}(10)$ & $-174.29(0.57)$ \\
\hline $\mathrm{N}(6)$ & $\mathrm{C}(9)$ & $\mathrm{C}(10)$ & $\mathrm{C}(11)$ & $-0.20(0.93)$ \\
\hline $\mathrm{N}(6)$ & $\mathrm{C}(9)$ & $\mathrm{C}(10)$ & $\mathrm{C}(101)$ & $-174.24(0.50)$ \\
\hline $\mathrm{C}(8)$ & $C(9)$ & $\mathrm{C}(10)$ & $\mathrm{C}(11)$ & $174.41(0.57)$ \\
\hline $\mathrm{C}(8)$ & $\mathrm{C}(9)$ & $\mathrm{C}(10)$ & $\mathrm{C}(101)$ & $0.37(0.88)$ \\
\hline C(9) & $\mathrm{C}(10)$ & $\mathrm{C}(11)$ & $\mathrm{N}(11)$ & $-0.53(0.94)$ \\
\hline $\mathrm{C}(9)$ & $\mathrm{C}(10)$ & $\mathrm{C}(11)$ & $C(12)$ & $-176.23(0.59)$ \\
\hline C(101) & $\mathrm{C}(10)$ & $\mathrm{C}(11)$ & $\mathrm{N}(11)$ & $173.62(0.50)$ \\
\hline C(101) & $\mathrm{C}(10)$ & $\mathrm{C}(11)$ & $\mathrm{C}(12)$ & $-2.08(0.87)$ \\
\hline C(9) & $\mathrm{C}(10)$ & $\mathrm{C}(101)$ & $\mathrm{C}(102)$ & $-72.92(0.73)$ \\
\hline C(9) & $C(10)$ & $C(101)$ & $C(106)$ & $107.70(0.63)$ \\
\hline $\mathrm{C}(11)$ & $\mathrm{C}(10)$ & $C(101)$ & $\mathrm{C}(102)$ & $112.41(0.63)$ \\
\hline $\mathrm{C}(11)$ & $\mathrm{C}(10)$ & $\mathrm{C}(101)$ & $C(106)$ & $-66.97(0.70)$ \\
\hline $\mathrm{N}(11)$ & $\mathrm{C}(11)$ & $\mathrm{C}(12)$ & $\mathrm{C}(13)$ & $0.27(0.70)$ \\
\hline $\mathrm{C}(10)$ & $\mathrm{C}(11)$ & $\mathrm{C}(12)$ & $C(13)$ & $176.51(0.59)$ \\
\hline $\mathrm{C}(11)$ & $\mathrm{C}(12)$ & $\mathrm{C}(13)$ & C(14) & $-0.40(0.72)$ \\
\hline $\mathrm{C}(12)$ & $\mathrm{C}(13)$ & $\mathrm{C}(14)$ & $\mathrm{N}(11)$ & $0.40(0.70)$ \\
\hline $\mathrm{C}(12)$ & $\mathrm{C}(13)$ & $\mathrm{C}(14)$ & C(15) & $-175.06(0.58)$ \\
\hline $\mathrm{N}(11)$ & $C(14)$ & $C(15)$ & $C(16)$ & $1.36(0.89)$ \\
\hline $\mathrm{N}(11)$ & $\mathrm{C}(14)$ & $\mathrm{C}(15)$ & $\mathrm{C}(151)$ & $-173.78(0.51)$ \\
\hline $\mathrm{C}(13)$ & $\mathrm{C}(14)$ & $\mathrm{C}(15)$ & $\mathrm{C}(16)$ & $176.04(0.56)$ \\
\hline $\mathrm{C}(13)$ & $\mathrm{C}(14)$ & $\mathrm{C}(15)$ & C(151) & $0.90(0.89)$ \\
\hline$C(14)$ & $\mathrm{C}(15)$ & $C(16)$ & $N(16)$ & $2.69(0.80)$ \\
\hline $\mathrm{C}(14)$ & $\mathrm{C}(15)$ & $\mathrm{C}(16)$ & $\mathrm{C}(17)$ & $-172.25(0.55)$ \\
\hline C(151) & $\mathrm{C}(15)$ & $C(16)$ & $\mathrm{N}(16)$ & $177.84(0.47)$ \\
\hline C(151) & $C(15)$ & $C(16)$ & $\mathrm{C}(17)$ & $2.89(0.87)$ \\
\hline$C(14)$ & $\mathrm{C}(15)$ & $C(151)$ & C(152) & $-70.71(0.74)$ \\
\hline $\mathrm{C}(14)$ & $\mathrm{C}(15)$ & $C(151)$ & C(156) & $110.67(0.63)$ \\
\hline$C(16)$ & $\mathrm{C}(15)$ & C(151) & C(152) & 113.99 ( 0.61) \\
\hline $\mathrm{C}(16)$ & $\mathrm{C}(15)$ & $C(151)$ & $C(156)$ & $-64.63(0.74)$ \\
\hline $\mathrm{N}(16)$ & $\mathrm{C}(16)$ & $\mathrm{C}(17)$ & $\mathrm{C}(18)$ & $-1.09(0.60)$ \\
\hline $\mathrm{C}(15)$ & $\mathrm{C}(16)$ & $\mathrm{C}(17)$ & C(18) & $174.35(0.55)$ \\
\hline$C(16)$ & $\mathrm{C}(17)$ & $\mathrm{C}(18)$ & C(19) & $0.15(0.63)$ \\
\hline C(17) & $\mathrm{C}(18)$ & C(19) & $\mathrm{N}(16)$ & $0.86(0.65)$ \\
\hline $\mathrm{C}(17)$ & $\mathrm{C}(18)$ & $\mathrm{C}(19)$ & $\mathrm{C}(1)$ & $-170.86(0.70)$ \\
\hline
\end{tabular}


Table of Torsional Angles in Degrees (cont)

for (tpfc)CrNTs

\begin{tabular}{|c|c|c|c|c|}
\hline Atom 1 & $\underline{\text { Atom }} \underline{2}$ & $\underline{\text { Atom }} \underline{3}$ & Atom 4 & Angle \\
\hline$S(20)$ & $\mathrm{C}(21)$ & $\mathrm{C}(22)$ & $\mathrm{C}(23)$ & $-178.61(0.48)$ \\
\hline$C(26)$ & $\mathrm{C}(21)$ & $\mathrm{C}(22)$ & $C(23)$ & $-0.43(0.93)$ \\
\hline S(20) & $\mathrm{C}(21)$ & $C(26)$ & $C(25)$ & $179.91(0.46)$ \\
\hline$C(22)$ & $\mathrm{C}(21)$ & $C(26)$ & $C(25)$ & $1.70(0.95)$ \\
\hline$C(21)$ & $\mathrm{C}(22)$ & $\mathrm{C}(23)$ & $\mathrm{C}(24)$ & $-1.76(0.96)$ \\
\hline $\mathrm{C}(22)$ & $C(23)$ & $C(24)$ & $C(25)$ & $2.55(0.98)$ \\
\hline$C(22)$ & C(23) & $C(24)$ & $C(27)$ & $-177.48(0.61)$ \\
\hline$C(23)$ & $\mathrm{C}(24)$ & $C(25)$ & $C(26)$ & $-1.16(0.94)$ \\
\hline $\mathrm{C}(27)$ & $C(24)$ & $C(25)$ & $C(26)$ & $178.87(0.60)$ \\
\hline $\mathrm{C}(24)$ & $C(25)$ & $C(26)$ & $C(21)$ & $-0.86(0.93)$ \\
\hline C(5) & $C(51)$ & C(52) & $\mathrm{F}(52)$ & $0.12(0.80)$ \\
\hline C(5) & $\mathrm{C}(51)$ & $C(52)$ & $C(53)$ & $-177.25(0.52)$ \\
\hline C(56) & $\mathrm{C}(51)$ & $\mathrm{C}(52)$ & $F(52)$ & $176.30(0.53)$ \\
\hline C(56) & $\mathrm{C}(51)$ & $C(52)$ & $C(53)$ & $-1.07(0.84)$ \\
\hline$C(5)$ & $C(51)$ & $C(56)$ & $F(56)$ & $-2.28(0.91)$ \\
\hline C(5) & $\mathrm{C}(51)$ & $C(56)$ & $C(55)$ & $177.70(0.59)$ \\
\hline C(52) & $\mathrm{C}(51)$ & $C(56)$ & $F(56)$ & $-178.46(0.56)$ \\
\hline C(52) & $\mathrm{C}(51)$ & $C(56)$ & C(55) & $1.52(0.93)$ \\
\hline $\mathrm{F}(52)$ & $C(52)$ & C(53) & $F(53)$ & $0.63(0.80)$ \\
\hline$F(52)$ & $\mathrm{C}(52)$ & C(53) & $C(54)$ & $-177.33(0.54)$ \\
\hline C(51) & $\mathrm{C}(52)$ & C(53) & $F(53)$ & $178.04(0.51)$ \\
\hline C(51) & C(52) & C(53) & $C(54)$ & $0.08(0.87)$ \\
\hline $\mathrm{F}(53)$ & C(53) & $C(54)$ & $F(54)$ & $1.51(0.91)$ \\
\hline$F(53)$ & C(53) & $C(54)$ & $C(55)$ & $-177.43(0.58)$ \\
\hline C(52) & C(53) & $\mathrm{C}(54)$ & $F(54)$ & $179.48(0.54)$ \\
\hline$C(52)$ & $C(53)$ & $C(54)$ & $C(55)$ & $0.54(0.96)$ \\
\hline$F(54)$ & C(54) & $C(55)$ & $\mathrm{F}(55)$ & $0.02(1.03)$ \\
\hline$F(54)$ & $C(54)$ & $C(55)$ & $C(56)$ & $-179.06(0.59)$ \\
\hline C(53) & C(54) & C(55) & $\mathrm{F}(55)$ & $178.97(0.61)$ \\
\hline C(53) & C(54) & C(55) & $C(56)$ & $-0.10(1.03)$ \\
\hline$F(55)$ & C(55) & $C(56)$ & $F(56)$ & $-0.08(1.07)$ \\
\hline $\mathrm{F}(55)$ & C(55) & $C(56)$ & $\mathrm{C}(51)$ & $179.95(0.58)$ \\
\hline C(54) & C(55) & $C(56)$ & $F(56)$ & $179.01(0.62)$ \\
\hline C(54) & C(55) & $C(56)$ & $C(51)$ & $-0.97(1.03)$ \\
\hline $\mathrm{C}(10)$ & $C(101)$ & $C(102)$ & $\mathrm{F}(102)$ & $-0.92(0.83)$ \\
\hline $\mathrm{C}(10)$ & $\mathrm{C}(101)$ & $\mathrm{C}(102)$ & C(103) & $-179.26(0.53)$ \\
\hline C(106) & $\mathrm{C}(101)$ & $C(102)$ & $\mathrm{F}(102)$ & $178.48(0.49)$ \\
\hline C(106) & $C(101)$ & $C(102)$ & C(103) & $0.14(0.84)$ \\
\hline $\mathrm{C}(10)$ & $C(101)$ & $C(106)$ & $F(106)$ & $-2.99(0.78)$ \\
\hline $\mathrm{C}(10)$ & $\mathrm{C}(101)$ & $C(106)$ & C(105) & $177.18(0.51)$ \\
\hline C(102) & $C(101)$ & $C(106)$ & $F(106)$ & $177.59(0.48)$ \\
\hline C(102) & $C(101)$ & $C(106)$ & C(105) & $-2.24(0.82)$ \\
\hline$F(102)$ & $C(102)$ & $C(103)$ & $F(103)$ & $0.03(0.80)$ \\
\hline$F(102)$ & $\mathrm{C}(102)$ & C(103) & C(104) & $-177.53(0.52)$ \\
\hline$C(101)$ & $C(102)$ & $C(103)$ & $F(103)$ & $178.40(0.51)$ \\
\hline C(101) & $C(102)$ & $C(103)$ & C(104) & $0.84(0.87)$ \\
\hline
\end{tabular}


Table of Torsional Angles in Degrees (cont)

for (tpfc)CrNTs

$\begin{array}{lllll}\underline{\text { Atom }} 1 & \text { Atom } 2 & \text { Atom } \underline{3} & \underline{\text { Atom }} \underline{4} & \underline{\text { Angle }} \\ \mathrm{F}(103) & \mathrm{C}(103) & \mathrm{C}(104) & \mathrm{F}(104) & 1.55(0.86) \\ \mathrm{F}(103) & \mathrm{C}(103) & \mathrm{C}(104) & \mathrm{C}(105) & -177.30(0.53) \\ \mathrm{C}(102) & \mathrm{C}(103) & \mathrm{C}(104) & \mathrm{F}(104) & 179.10(0.52) \\ \mathrm{C}(102) & \mathrm{C}(103) & \mathrm{C}(104) & \mathrm{C}(105) & 0.25(0.89) \\ \mathrm{F}(104) & \mathrm{C}(104) & \mathrm{C}(105) & \mathrm{F}(105) & 0.99(0.84) \\ \mathrm{F}(104) & \mathrm{C}(104) & \mathrm{C}(105) & \mathrm{C}(106) & 178.86(0.52) \\ \mathrm{C}(103) & \mathrm{C}(104) & \mathrm{C}(105) & \mathrm{F}(105) & 179.84(0.52) \\ \mathrm{C}(103) & \mathrm{C}(104) & \mathrm{C}(105) & \mathrm{C}(106) & -2.30(0.87) \\ \mathrm{F}(105) & \mathrm{C}(105) & \mathrm{C}(106) & \mathrm{F}(106) & 1.42(0.77) \\ \mathrm{F}(105) & \mathrm{C}(105) & \mathrm{C}(106) & \mathrm{C}(101) & -178.74(0.49) \\ \mathrm{C}(104) & \mathrm{C}(105) & \mathrm{C}(106) & \mathrm{F}(106) & -176.46(0.50) \\ \mathrm{C}(104) & \mathrm{C}(105) & \mathrm{C}(106) & \mathrm{C}(101) & 3.38(0.86) \\ \mathrm{C}(15) & \mathrm{C}(151) & \mathrm{C}(152) & \mathrm{F}(152) & 2.91(0.81) \\ \mathrm{C}(15) & \mathrm{C}(151) & \mathrm{C}(152) & \mathrm{C}(153) & -179.48(0.53) \\ \mathrm{C}(156) & \mathrm{C}(151) & \mathrm{C}(152) & \mathrm{F}(152) & -178.40(0.49) \\ \mathrm{C}(156) & \mathrm{C}(151) & \mathrm{C}(152) & \mathrm{C}(153) & -0.79(0.82) \\ \mathrm{C}(15) & \mathrm{C}(151) & \mathrm{C}(156) & \mathrm{F}(156) & -3.01(0.80) \\ \mathrm{C}(15) & \mathrm{C}(151) & \mathrm{C}(156) & \mathrm{C}(155) & 177.46(0.52) \\ \mathrm{C}(152) & \mathrm{C}(151) & \mathrm{C}(156) & \mathrm{F}(156) & 178.29(0.47) \\ \mathrm{C}(152) & \mathrm{C}(151) & \mathrm{C}(156) & \mathrm{C}(155) & -1.24(0.82) \\ \mathrm{F}(152) & \mathrm{C}(152) & \mathrm{C}(153) & \mathrm{F}(153) & -1.82(0.84) \\ \mathrm{F}(152) & \mathrm{C}(152) & \mathrm{C}(153) & \mathrm{C}(154) & 179.65(0.54) \\ \mathrm{C}(151) & \mathrm{C}(152) & \mathrm{C}(153) & \mathrm{F}(153) & -179.43(0.52) \\ \mathrm{C}(151) & \mathrm{C}(152) & \mathrm{C}(153) & \mathrm{C}(154) & 2.05(0.91) \\ \mathrm{F}(153) & \mathrm{C}(153) & \mathrm{C}(154) & \mathrm{F}(154) & -0.09(0.87) \\ \mathrm{F}(153) & \mathrm{C}(153) & \mathrm{C}(154) & \mathrm{C}(155) & -179.78(0.54) \\ \mathrm{C}(152) & \mathrm{C}(153) & \mathrm{C}(154) & \mathrm{F}(154) & 178.43(0.54) \\ \mathrm{C}(152) & \mathrm{C}(153) & \mathrm{C}(154) & \mathrm{C}(155) & -1.26(0.92) \\ \mathrm{F}(154) & \mathrm{C}(154) & \mathrm{C}(155) & \mathrm{F}(155) & -0.94(0.85) \\ \mathrm{F}(154) & \mathrm{C}(154) & \mathrm{C}(155) & \mathrm{C}(156) & 179.56(0.53) \\ \mathrm{C}(153) & \mathrm{C}(154) & \mathrm{C}(155) & \mathrm{F}(155) & 178.74(0.53) \\ \mathrm{C}(153) & \mathrm{C}(154) & \mathrm{C}(155) & \mathrm{C}(156) & -0.76(0.89) \\ \mathrm{F}(155) & \mathrm{C}(155) & \mathrm{C}(156) & \mathrm{F}(156) & 3.02(0.80) \\ \mathrm{F}(155) & \mathrm{C}(155) & \mathrm{C}(156) & \mathrm{C}(151) & -177.45(0.51) \\ \mathrm{C}(154) & \mathrm{C}(155) & \mathrm{C}(156) & \mathrm{F}(156) & -177.49(0.51) \\ \mathrm{C}(154) & \mathrm{C}(155) & \mathrm{C}(156) & \mathrm{C}(151) & 2.04(0.88) \\ & & & & \end{array}$




\section{References.}

${ }^{1}$ Meier-Callahan, A. E.; Di Bilio, A. J.; Simkhovich, L.; Mahammed, A.; Goldberg, I.; Gray, H. B.; Gross, Z. Inorg. Chem. 2001, 40, 6788.

${ }^{2}$ Nakamoto, K. Infrared Spectra of Inorganic and Coordination Compounds, $2^{\text {nd }}$ Ed. WileyInterscience: New York, NY. 1970. p. 224.

${ }^{3}$ (a) Evans, D. F. J. Chem. Soc. 1959, 2003. (b) Grant, D. H. J. Chem. Ed. 1995, 72, 39.

(c) Schubert, E. M. J. Chem. Ed. 1992, 69, 62. 
Appendix Derivation of $\frac{K_{\mathrm{eq}}[\mathrm{EtOAc}][\mathrm{Mn}(\mathrm{tpfc})]_{0}}{1+K_{\mathrm{eq}}[\mathrm{EtOAc}]}=[\mathrm{Mn}(\mathrm{tpfc})$ EtOAc $]$

Note: It is assumed that the added Ethyl acetate is in great excess to the other concentrations.

Therefore, $[$ EtOAc $]=[\text { EtOAc }]_{0}$

$$
\begin{aligned}
& \mathrm{Mn}(\mathrm{tpfc})+\text { EtOAc } \stackrel{K_{\mathrm{eq}}}{\rightleftharpoons} \mathrm{Mn}(\mathrm{tpfc}) \text { EtOAc } \\
& K_{\mathrm{eq}}=\frac{[\mathrm{Mn}(\mathrm{tpfc}) \text { EtOAc }]}{[\mathrm{Mn}(\mathrm{tpfc})][\text { EtOAc }]}
\end{aligned}
$$

$\frac{[\mathrm{Mn}(\mathrm{tpfc}) \mathrm{EtOAc}]}{[\mathrm{Mn}(\mathrm{tpfc})]}=K_{\mathrm{eq}}[\mathrm{EtOAc}]$

Since $[M n(t p f c)]_{0}=[M n(t p f c)]+[M n(t p f c) E t O A c]$,

$\frac{[\mathrm{Mn}(\mathrm{tpfc}) \text { EtOAc }]}{[\mathrm{Mn}(\mathrm{tpfc})]_{0}-[\mathrm{Mn}(\mathrm{tpfc}) \text { EtOAc }]}=K_{\text {eq }}[$ EtOAc $]$

Dividing top and bottom by [Mn(tpfc)EtOAc]

$$
\frac{1}{\frac{[\mathrm{Mn}(\mathrm{tpfc})]_{0}}{[\mathrm{Mn}(\mathrm{tpfc}) \mathrm{EtOAc}]}-1}=K_{\mathrm{eq}}[\text { EtOAc }]
$$

Rearranging gives

$$
\begin{aligned}
& \frac{1}{K_{\mathrm{eq}}[\text { EtOAc }]}+1=\frac{[\mathrm{Mn}(\mathrm{tpfc})]_{0}}{[\mathrm{Mn}(\mathrm{tpfc}) \text { EtOAc }]} \\
& \frac{\frac{1}{K_{\mathrm{eq}}[\text { EtOAc }]}+1}{[\mathrm{Mn}(\mathrm{tpfc})]_{0}}=\frac{1}{[\mathrm{Mn}(\mathrm{tpfc}) \text { EtOAc }]} \\
& \frac{1}{K_{\mathrm{eq}}[\text { EtOAc }][\mathrm{Mn}(\mathrm{tpfc})]_{0}}+\frac{1}{[\mathrm{Mn}(\mathrm{tpfc})]_{0}}=\frac{1}{[\mathrm{Mn}(\mathrm{tpfc}) \text { EtOAc }]}
\end{aligned}
$$

Reciprocating both sides

$$
\begin{aligned}
& \frac{1}{\frac{1}{K_{\text {eq }}[\text { EtOAc }][\mathrm{Mn}(\mathrm{tpfc})]_{0}}+\frac{1}{[\mathrm{Mn}(\mathrm{tpfc})]_{0}}}=[\mathrm{Mn}(\mathrm{tpfc}) \text { EtOAc }] \\
& \frac{1}{\frac{1}{K_{\mathrm{eq}}[\text { EtOAc }][\mathrm{Mn}(\mathrm{tpfc})]_{0}}+\frac{K_{\mathrm{eq}}[\text { EtOAc }]}{K_{\mathrm{eq}}[\text { EtOAc }][\mathrm{Mn}(\mathrm{tpfc})]_{0}}}=[\mathrm{Mn}(\mathrm{tpfc}) \text { EtOAc }]
\end{aligned}
$$




$\frac{1}{\frac{1}{K_{\text {eq }}[\text { EtOAc }][\mathrm{Mn}(\mathrm{tpfc})]_{0}}+\frac{K_{\mathrm{eq}}[\text { EtOAc }]}{K_{\mathrm{eq}}[\text { EtOAc }][\mathrm{Mn}(\mathrm{tpfc})]_{0}}}=[\mathrm{Mn}(\mathrm{tpfc})$ EtOAc $]$
$\frac{1}{\frac{1+K_{\mathrm{eq}}[\mathrm{EtOAc}]}{K_{\mathrm{eq}}[\text { EtOAc }][\mathrm{Mn}(\mathrm{tpfc})]_{0}}}=[\mathrm{Mn}(\mathrm{tpfc})$ EtOAc $]$
$\frac{K_{\mathrm{eq}}[\text { EtOAc }][\mathrm{Mn}(\mathrm{tpfc})]_{0}}{1+K_{\mathrm{eq}}[\text { EtOAc }]}=[\mathrm{Mn}(\mathrm{tpfc})$ EtOAc $]$

